\title{
2-D measurements of plasma electron density using coherence imaging with a pixelated phase mask
}

\author{
J. S. Allcock, ${ }^{1,2}$, a) S. A. Silburn, ${ }^{2}$ R. M. Sharples, ${ }^{1}$ J. R. Harrison, ${ }^{2}$ N. J. Conway, ${ }^{2}$ and J. W. M. Vernimmen ${ }^{3}$ \\ ${ }^{1)}$ Centre for Advanced Instrumentation, Department of Physics, Durham University, Durham, DH1 3LE, \\ $U K$ \\ ${ }^{2)}$ Culham Centre for Fusion Energy, Culham Science Centre, Abingdon, Oxon, OX14 3DB, \\ $U K$ \\ ${ }^{3)}$ DIFFER - Dutch Institute for Fundamental Energy Research, De Zaale 20, 5612 AJ Eindhoven, \\ the Netherlands
}

(Dated: 26 October 2021)

\begin{abstract}
In this paper, the pixelated phase mask (PPM) method of interferometry is applied to coherence imaging $(\mathrm{CI})$ - a passive, narrowband spectral imaging technique for diagnosing the edge and divertor regions of fusion plasma experiments. Compared to previous CI designs which use a linear phase mask, the PPM method allows for a higher possible spatial resolution. The PPM method is also observed to give a higher instrument contrast (analogous to a more narrow spectrometer instrument function). A single-delay PPM instrument is introduced, as well as a multi-delay system which uses a combination of both pixelated and linear phase masks to encode the coherence of the observed radiation at four different interferometer delays simultaneously. The new methods are demonstrated with measurements of electron density $n_{\mathrm{e}}$, via Stark broadening of the $\mathrm{H}_{\gamma}$ emission line at $434.0 \mathrm{~nm}$, made on the Magnum-PSI linear plasma experiment. Comparison of the Abel-inverted multi-delay CI measurements with Thomson scattering shows agreement across the $3 \times 10^{19} \mathrm{~m}^{-3}<n_{\mathrm{e}}<1 \times 10^{21} \mathrm{~m}^{-3}$ range. For the single-delay CI results, agreement is found for $n_{\mathrm{e}}>1 \times 10^{20} \mathrm{~m}^{-3}$ only. Accurate and independent interpretation of single-delay CI data at lower $n_{\mathrm{e}}$ was not possible due to Doppler broadening and continuum emission.
\end{abstract}

\section{INTRODUCTION}

Producing steady-state fusion power in the core of a magnetically confined plasma while keeping the power load to the divertor target below the erosion limit is known as the 'heat exhaust problem' ${ }^{1}$. Solutions to this problem will likely include maintaining the divertor in a detached state and with an optimized geometry for both the machine and the magnetic field. When it begins operations, the MAST Upgrade spherical tokamak will test a range of novel divertor configurations, aided by a suite of diagnostics "designed for as high space and time resolution as is currently feasible" 2 .

One of these diagnostics is coherence imaging (CI) - a narrowband spectral imaging technique that measures spectral line emission from the plasma edge and provides 2-D images of the lower-order moments of the observed spectral distribution (broadly, the brightness, shift and width of the line $)^{3-5}$. The CI instrument is a common-path polarization interferometer ${ }^{6}$ and the spectrum is encoded in the interference fringe pattern according to the principles of Fourier transform spectroscopy ${ }^{7}$. Without the need for an entrance slit, CI can achieve better spatial coverage than is typically practical for grating spectrometers. Many CI designs are also 'snapshot' in that they modulate the interferometer delay spatially across the sensor, so the time resolution is limited only by the camera frame rate or the brightness of the emission. Two CI instruments are planned for MAST-U, one standalone and the other occupying a channel in a multi-wavelength imaging (MWI) system ${ }^{8}$. One CI application of interest on MAST-U is the measurement of impurity ion flow velocity in the plasma edge, which is well established ${ }^{9-14}$. A second ap-

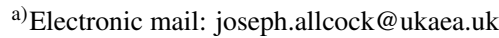

plication of interest has received less attention: the measurement of electron density $n_{\mathrm{e}}$ in the divertor via Stark broadening of hydrogen Balmer emission. While this application is established for grating spectrometers ${ }^{15-19}$, for CI it has been demonstrated at the proof-of-concept level only ${ }^{20}$.

Measurements of $n_{\mathrm{e}}$ are routinely made on fusion experiments using Thomson scattering, interferometry and Langmuir probes ${ }^{19}$. However, each of these techniques has limited spatial coverage: Thomson scattering measurements are localized along the path of a laser beam, interferometry is typically limited to a single line-of-sight integral value and Langmuir probes are restricted to the very edge of the plasma. CI itself is limited to measuring regions where the neutral hydrogen density is high enough for intense Balmer line emission - the scrape-off layer and divertor — but within these regions CI could achieve better coverage than the existing techniques. This would allow for a more complete comparison with physics simulations.

In this work we present CI measurements of $n_{\mathrm{e}}$ made on the Magnum-PSI linear plasma experiment ${ }^{21}$ in conditions relevant to the study of tokamak divertor physics $\left(n_{\mathrm{e}} \sim 10^{20} \mathrm{~m}^{-3}\right.$ and temperature $T_{\mathrm{e}} \sim 1 \mathrm{eV}$ ). The motivation for the work is to test several new CI techniques before their application to measurements of $n_{\mathrm{e}}$ in the MAST-U divertor. The main novelty here is the demonstration of two new CI instrument configurations, both of which incorporate a pixelated micro-polarizer array, generating a pixelated fringe pattern that maximizes the spatial resolution of the measurement. This is based on an existing technique in interferometry called pixelated phase mask (PPM) interferometry. Another significant focus of this work is in CI data analysis, for which we make use of state-of-theart Balmer lineshape calculations and Bayesian curve-fitting in the inference of $n_{\mathrm{e}}$. The resulting $n_{\mathrm{e}}$ profiles are benchmarked against Magnum-PSI's Thomson scattering diagnostic across a wide range of $n_{\mathrm{e}}$. 


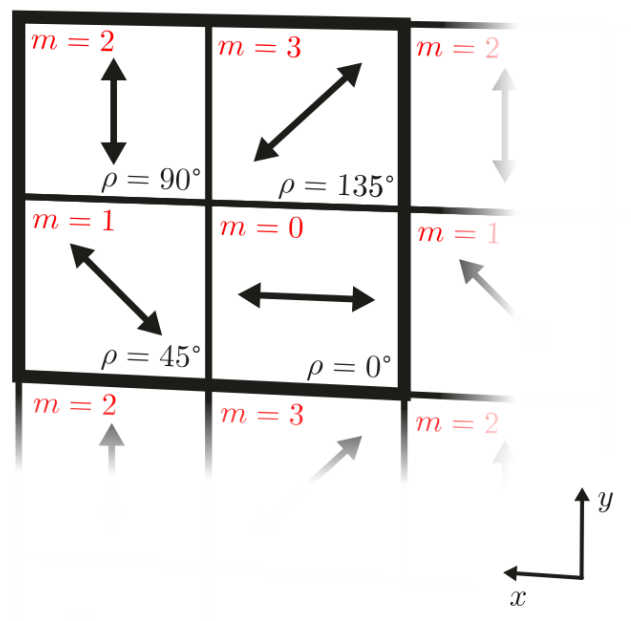

FIG. 1. Pixelated polarizers arranged in the repeating $2 \times 2$ grid pattern of the Sony IMX250MZR CMOS sensor? used in this work. Pixelated polarizer orientations are identified by index $m$ as shown.

The rest of the paper proceeds as follows: Section II introduces the PPM CI technique. In Section III, the application of CI to measurement of $n_{\mathrm{e}}$ is discussed. Section IV presents experimental CI measurements of $n_{\mathrm{e}}$ made on the Magnum-PSI experiment and finally the conclusions are given in Section V.

\section{COHERENCE IMAGING WITH A PIXELATED PHASE MASK}

Polarized sensors have an array of pixelated wire-grid polarizers bonded directly to the chip at different orientations. Figure 1 shows the repeating $2 \times 2$ polarizer layout of the sensor used in this work. Each $2 \times 2$ pixel grid in the image encodes the first three Stokes parameters of an observed scene, from which the degree of linear polarization and the angle of polarization can be calculated. The use of polarized sensors in interferometry was proposed by Millerd et al. ${ }^{22}$ in 2004 with pixelated phase mask (PPM) interferometry. Compared to a linear phase mask (LPM), which produces sinusoidal fringes that are roughly straight and parallel (see e.g. Figure 1 in Silburn et al. ${ }^{10}$ ), the PPM technique has a more compact design and a phase mask that is fixed on manufacture instead of being dependant on the alignment of the optics. The spatial resolution of a PPM instrument is higher than is possible for an LPM instrument using an otherwise equivalent sensor. This advantage is discussed in more detail in Section II A. The PPM technique has been demonstrated for imaging of biological specimens ${ }^{23,24}$ and of sound waves ${ }^{25}$. In this section we will consider how PPM interferometry can be used for coherence imaging of plasma experiments.

\section{A. Single-delay configuration}

\section{Measurement principle}

In a simple polarization interferometer, a birefringent waveplate is sandwiched between two polarizers, with the wave-

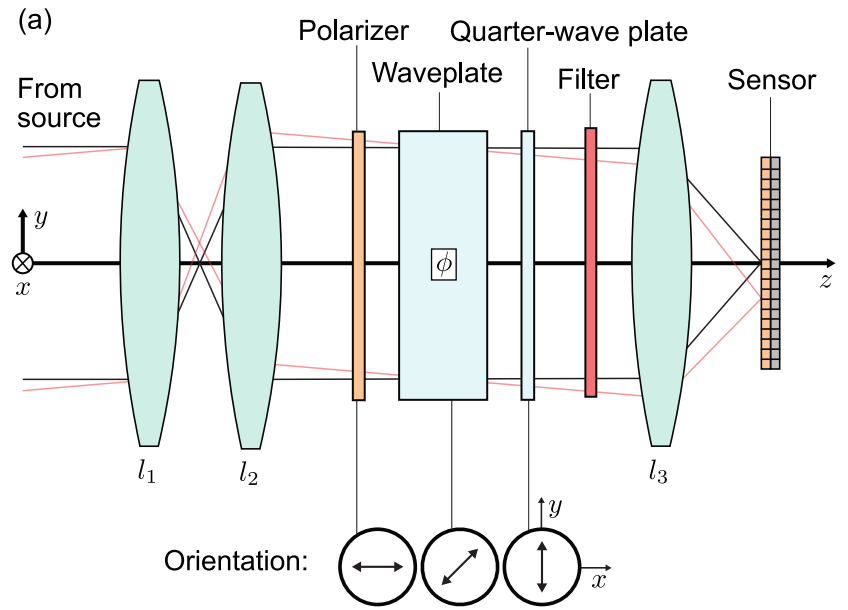

(b)

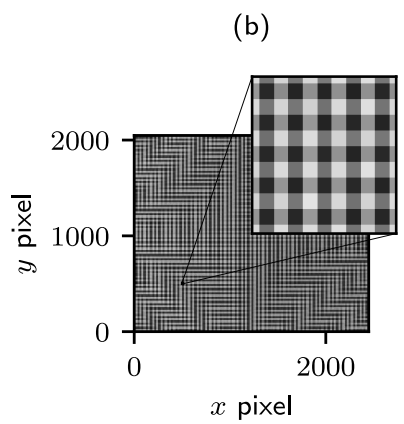

(c)
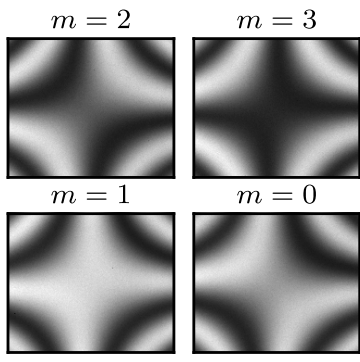

(d) Measured $\Phi$

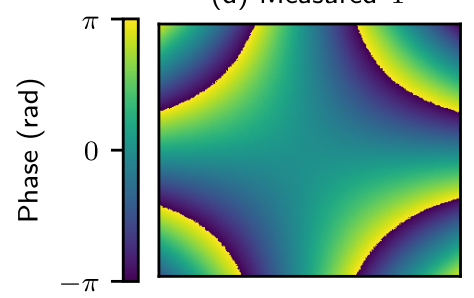

(e) Modeled $\Phi$

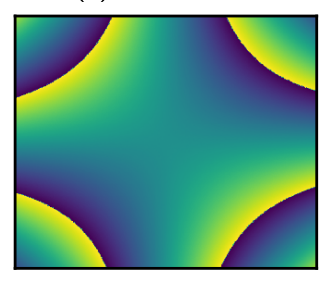

FIG. 2. (a) Optical layout for the single-delay CI configuration. (b) A raw, uniform-brightness calibration image captured by this instrument observing isolated Cd I line emission at $467.8 \mathrm{~nm}$. (c) The four images corresponding to the different orientations of pixelated polarizer shown separately. (d) Demodulated interferogram phase $\Phi\left(\phi_{0}\right)$ extracted from the raw calibration image in (b). (e) The corresponding modeled phase image.

plate's optic axis making an angle of $45^{\circ}$ with the transmission axes of the polarizers ${ }^{6,26}$. The waveplate resolves the light into two equal-amplitude beams in orthogonal polarization states and imparts a phase delay between them. By introducing a quarter-wave plate (QWP) between the waveplate and the final polarizer, with fast axis orthogonal to the front polarizer, the interferometer delay is now determined by the orientation of the final polarizer ${ }^{22,27}$. As such, substituting a polarized sensor for the final polarizer results in as many samples of the interference pattern (over a $2 \pi$ rad range of interferometer delays) as there are unique polarizer orientations. Figure 2(a) shows a schematic of the setup described, with a bandpass optical filter and imaging lens between the QWP and polarized sensor. This three-lens optical layout shown, with an intermediate image between $l_{1}$ and $l_{2}$, is based on the MAST CI system ${ }^{9,10}$.

To model the observed interferogram, we use Mueller ma- 
trices and Stokes vectors ${ }^{28}$. Let $\mathrm{M}_{\mathrm{P}}(\rho)$ be the Mueller matrix for a polarizer whose transmission axis makes an angle $\rho$ with the $x$-axis. Let $\mathrm{M}_{\mathrm{QWP}}(\rho)$ and $\mathrm{M}_{\mathrm{LR}}(\rho, \phi)$ be the Mueller matrices for a QWP and general linear retarder respectively, whose fast axes make an angle $\rho$ with the $x$-axis. The phase delay in radians imparted by the retarder $\phi$ has an implicit dependence on the light's frequency $v$. See Appendix A for explicit matrix definitions. The total Mueller matrix for the system as described is then

$$
\mathrm{M}_{\mathrm{SD}} \equiv \mathrm{M}_{\mathrm{P}}\left(m \frac{\pi}{4}\right) \mathrm{M}_{\mathrm{QWP}}\left(\frac{\pi}{2}\right) \mathrm{M}_{\mathrm{LR}}\left(\frac{\pi}{4}, \phi\right) \mathrm{M}_{\mathrm{P}}(0) .
$$

To represent the polarized sensor, the orientation of the final polarizer is written in terms of pixel number $m$, with reference to Figure 1. The Stokes vector representing incident, unpolarized light can be written $S(v)=(I(v), 0,0,0)$ where $I(v)$ is the total spectral radiance. The Stokes vector representing light reaching the sensor is then

$$
S^{\prime}(v)=\left(\begin{array}{c}
I^{\prime}(v) \\
Q^{\prime}(v) \\
U^{\prime}(v) \\
V^{\prime}(v)
\end{array}\right)=\mathrm{M}_{\mathrm{SD}}(v) \mathrm{S}(v)
$$

where the $v$-dependence of the Mueller matrix has now been made explicit. The radiance at the sensor is $I^{\prime} \equiv \int_{-\infty}^{\infty} I^{\prime}(v) d v$, which can be evaluated to give:

$$
I^{\prime}=\frac{I}{4}\left(1+\Re\left\{\gamma\left(\phi_{0}+m \frac{\pi}{2}\right)\right\}\right),
$$

where we have also defined $I \equiv \int_{-\infty}^{\infty} I(v) d v$. Here, $\gamma\left(\phi_{0}\right)$ is the complex degree of temporal coherence. It is a function of the phase delay $\phi_{0}$ between the two interferometer beams at frequency $v_{0}$, corresponding to the center-of-mass frequency of the observed (narrowband) spectrum. The Wiener-Khinchin theorem relates $\gamma\left(\phi_{0}\right)$ to the area-normalized spectral distribution $g(v) \equiv I(v) / I$ by Fourier transform ${ }^{26}$, which can be written as:

$$
\gamma\left(\phi_{0}\right) \approx \int_{-\infty}^{\infty} g(v) \exp \left(i \phi_{0}\left[1+\kappa_{0}\left(\frac{v-v_{0}}{v_{0}}\right)\right]\right) d v .
$$

Here, $\kappa_{0}$ is a dimensionless parameter of order 1 that provides a first-order approximation of the instrument dispersion ${ }^{5,29}$. From Equation 3 we can see that each $2 \times 2$ pixel sub-array samples $\gamma$ at $\frac{\pi}{2}$ rad delay intervals, thereby encoding information about $g(v)$ in a pixelated interference pattern.

The CI technique relies on $g(v)$ being entirely determined by a small number well-understood physics processes in the emitting plasma. That way, the fringe contrast $\zeta \equiv|\gamma|$ and fringe phase $\Phi \equiv \arg \gamma$ need only be observed at a small number of interferometer delays to recover the parameter(s) of interest. The waveplate thickness, determining delay $\phi_{0}$, is chosen so as to maximize sensitivity to changes in those parameters. Generally, this means setting the delay time to be $\frac{\phi_{0}}{2 \pi v_{0}} \sim \tau$ where $\tau$ is the coherence time of the radiation.

\section{Hardware}

The hardware used in this work is the MAST CI system ${ }^{9,10}$, modified to accommodate the FLIR Blackfly $\mathrm{S}$ machine vision camera $^{30}$, which incorporates the polarized 12-bit CMOS sensor shown in Figure 1. The sensor format is $2448 \times 2048$ and the pixel dimension is $3.45 \mu \mathrm{m}$. Pixelated polarizers have a low extinction ratio $E$ when compared to other available polarizers, which leads to a multiplicative fringe contrast degradation factor ${ }^{9}$, calculated as $\zeta_{\mathrm{P}}=(E-1) /(E+1)$. For the sensor used in this work, and at the wavelengths considered, $E \approx 400$, giving $\zeta_{\mathrm{P}} \approx 0.995$. The three digital single-lens reflex (DSLR) lenses $\left(l_{1}, l_{2}\right.$ and $l_{3}$, with reference to Figure 2(a)) used in this section, have focal lengths $70 \mathrm{~mm}, 105 \mathrm{~mm}$ and $150 \mathrm{~mm}$ respectively. DSLR lenses were originally chosen for the MAST CI system as they are optimized for high-quality, wide-angle imaging ${ }^{9}$. Three waveplates were available for use in this work. They are each made from alpha barium borate $\left(\alpha\right.$-BBO) and have measured thicknesses $L_{\mathrm{WP}}=4.48 \mathrm{~mm}$, $6.35 \mathrm{~mm}$ and $9.79 \mathrm{~mm}$, each value $\pm 0.02 \mathrm{~mm}$. The zeroorder QWP used is a polymer retarder film. All interferometer components were mounted inside a temperature-stabilized cell with a nominal regulation accuracy of $\pm 0.25^{\circ} \mathrm{C}$.

A uniform-brightness test image was obtained with the CI instrument in the single-delay PPM configuration shown in Figure 2(a), with a Cd gas-discharge lamp illuminating an integrating sphere as the light source. The Cd I line at $467.8 \mathrm{~nm}$ was isolated using a bandpass filter. For demonstration purposes, all three available waveplates were used at once, their optic axes aligned so as to combine the phase delays constructively. The resulting image is shown in Figure 2(b). In Figure 2(c) the four interleaved images corresponding to the four polarizer orientations are shown separately. The hyperbolic fringe pattern is caused by the dependence of $\phi_{0}$ on ray angle through the waveplate ${ }^{31}$ and the $\frac{\pi}{2}$ rad phase shift between images of consecutive $m$ is clear.

\section{Demodulation}

Interferogram contrast $\zeta \equiv|\gamma|$ and phase $\Phi \equiv \arg \gamma$ are related to the observed spectrum $g(v)$ by Equation 4 . By writing $\gamma\left(\phi_{0}\right)$ as $\zeta \exp (i \Phi)$, we can rewrite Equation 3 as

$$
\begin{aligned}
& I_{0}^{\prime}=\frac{I}{4}(1+\zeta \cos \Phi) \\
& I_{1}^{\prime}=\frac{I}{4}(1-\zeta \sin \Phi) \\
& I_{2}^{\prime}=\frac{I}{4}(1-\zeta \cos \Phi) \\
& I_{3}^{\prime}=\frac{I}{4}(1+\zeta \sin \Phi),
\end{aligned}
$$

where the expression for each of the four polarizer orientations has been written out explicitly, the subscripts denoting pixel number $m$. Different PPM demodulation algorithms have been suggested ${ }^{22,32,33}$ for recovering the $\zeta$ and $\Phi$ images from this kind of interferogram, but in this work we use the simple 'four-bucket' algorithm ${ }^{22}$. The brightness image is recovered as

$$
I \approx \sum_{m=0}^{3} I_{m}^{\prime}
$$

the phase image is recovered as

$$
\Phi \approx \arctan \left(\frac{I_{3}^{\prime}-I_{1}^{\prime}}{I_{0}^{\prime}-I_{2}^{\prime}}\right)
$$


and the contrast image is recovered as

$$
\zeta \approx \frac{1}{I} \sqrt{8 \sum_{m=0}^{3}\left(I_{m}^{\prime}-\frac{I}{4}\right)^{2}} .
$$

Figure 2(d) shows the demodulated $\Phi$ from the calibration image and Figure 2(e) shows the corresponding $\Phi$ modeled using Equation 12 from Veiras et al. ${ }^{31}$. Since the model parameter values are not sufficiently accurate to recover absolute $\Phi$, it is set to zero at the image center in Figures 2(d) and (e).

For typical CI instruments that use an LPM design, it is necessary to shear the delay across the image in one direction. The required fixed delay and shear can both be produced using a 'displacer plate'12,13,34 or else produced separately using a waveplate and Savart plate in combination ${ }^{9,10,14}$. A displacer plate is a single birefringent plate with an intermediate cut angle (i.e. with its optic axis neither parallel to nor perpendicular to the front surface of the plate) and a Savart plate is a composite of two displacer plates, aligned so as to produce the shear while imparting no net delay for on-axis rays ${ }^{6,26}$.

The spatial resolution at which $\zeta$ and $\Phi$ are recovered from an LPM interferogram is anisotropic. Perpendicular to the direction of phase shear, it is set by the pixel width (assuming detector-limited operation). Parallel to the direction of phase shear, the spatial resolution is worse, depending on the fringe frequency and the width of the Fourier-domain filter used in the demodulation process. Modeling carried out in previous work $^{9}$ of MAST CI images suggests 2 fringe periods as a typical spatial resolution in this direction. The LPM fringe period is typically chosen to be $>6$ pixels, to avoid contrast degradation due to phase shear across each pixel's collection area. The PPM spatial resolution is isotropic and is 2 pixels or better in both dimensions, depending on the demodulation algorithm used $^{32}$.

\section{B. Multi-delay configuration}

Plasma line spectra are often complicated, with multiple components, multiple broadening mechanisms and a continuum background. In such cases, encoding $\gamma$ at multiple interferometer delays can make interpretation easier and reduce systematic error. For example, Michael et al. ${ }^{29}$ used CI measurements made at multiple delays to characterize the neutral velocity distribution function in an argon plasma via the Doppler-broadened lineshape. That work used a single-delay CI design, with samples of $\gamma$ built up by observing repeated plasma discharges with different waveplate thicknesses. More recent work ${ }^{35}$ made measurements of charge exchange recombination emission using a snapshot multi-delay CI design, but interpretation of the results was inconclusive.

\section{Measurement principle}

The single-delay CI system from the previous section can be turned into a multi-delay system by introducing a polarizer, waveplate and Savart plate to the front of the interferometer, with order and orientations shown in Figure 3(a). The Mueller matrix for this configuration can be written:

$$
\mathrm{M}_{\mathrm{MD}} \equiv \mathrm{M}_{\mathrm{SD}} \mathrm{M}_{\mathrm{LR}}\left(\frac{\pi}{4}, \psi\right) \mathrm{M}_{\mathrm{P}}(0)
$$

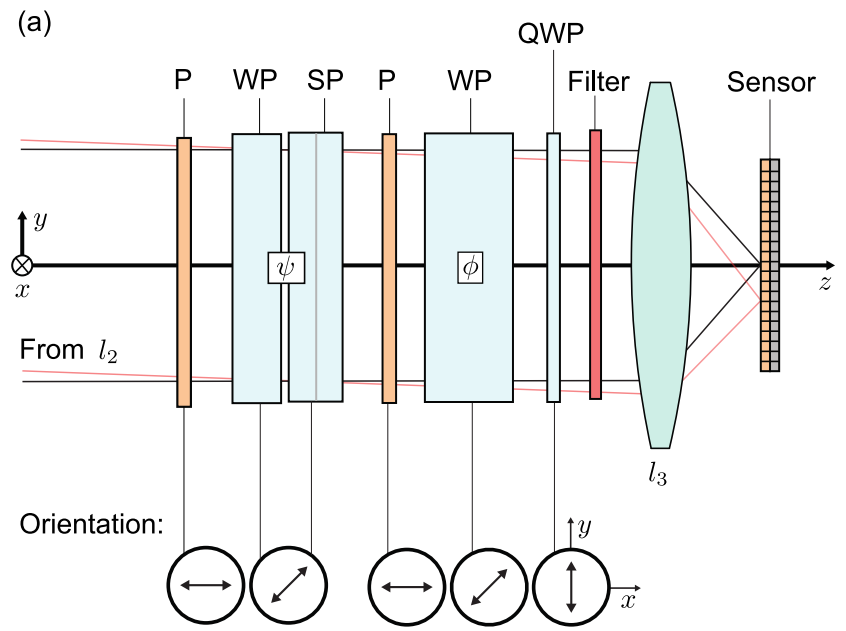

(b)

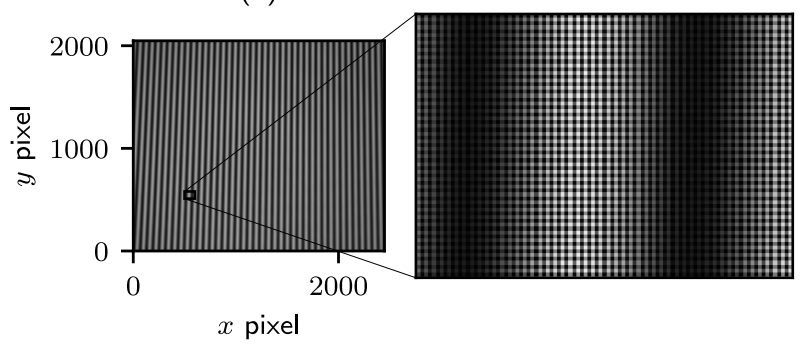

(d)
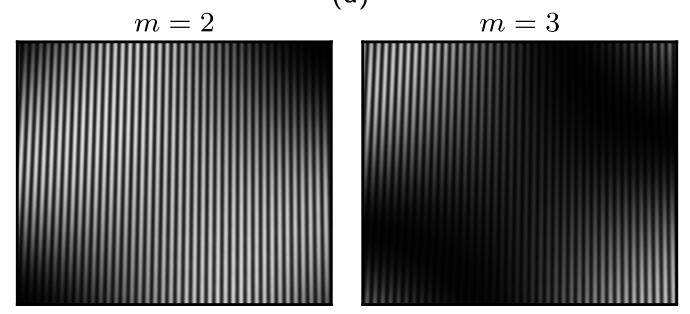

$m=1$

$m=0$
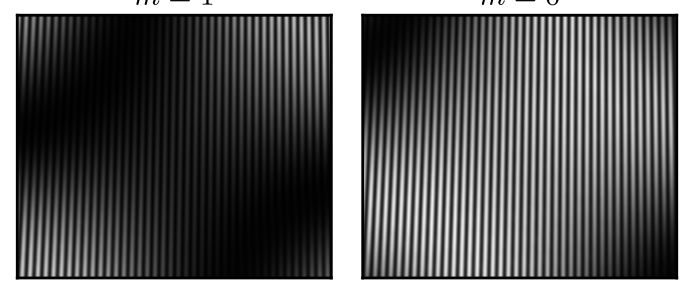

FIG. 3. (a) Optical layout for multi-delay coherence imaging. The system's front two lenses, $l_{1}$ and $l_{2}$, are not shown here. The shorthand labels here refer to polarizer (P), waveplate (WP), Savart plate (SP) and quarter-wave plate (QWP). (b) A raw, uniform-brightness calibration image captured by this instrument observing isolated $\mathrm{Cd}$ I line emission at $467.8 \mathrm{~nm}$. (c) A zoomed view of (b). (d) The four images corresponding to the different orientations of pixelated polarizer, shown separately.

Where $\mathrm{M}_{\mathrm{SD}}$ was defined in Equation 1 and where $\psi$ is the delay imparted by a waveplate and Savart plate in combination, with implicit dependence on frequency and sensor plane position. Instead of this waveplate-Savart plate combination, a displacer plate could be used. To find an expression for the radiance at the sensor plane $I^{\prime}$ under observation of unpolarized light we proceed as in the case of the single-delay configuration by evaluating $\int_{-\infty}^{\infty} \mathrm{M}_{\mathrm{MD}}(v) \mathrm{S}(v) d v$. It can be shown that 
this gives:

$$
\begin{aligned}
I^{\prime}=\frac{I}{8}( & +\Re\left\{\gamma\left(\psi_{0}\right)\right\} \\
& +\Re\left\{\gamma\left(\phi_{0}+m \frac{\pi}{2}\right)\right\} \\
& +\frac{1}{2} \mathfrak{R}\left\{\gamma\left(\phi_{0}+\psi_{0}+m \frac{\pi}{2}\right)\right\} \\
& \left.+\frac{1}{2} \mathfrak{R}\left\{\gamma\left(\phi_{0}-\psi_{0}+m \frac{\pi}{2}\right)\right\}\right) .
\end{aligned}
$$

Here, $\psi_{0}$ is the phase delay imparted by the waveplate and Savart plate in combination at centre-of-mass frequency $v_{0}$. We can see that this interferogram encodes $\gamma$ at four fixed delays: $\phi_{0}, \psi_{0}, \phi_{0}+\psi_{0}$ and $\phi_{0}-\psi_{0}$. These four delays arise as follows. Light exiting the second polarizer in the system is the sum of two beams with relative delay $\psi_{0}$ between them. The second waveplate then splits each of these beams again into a pair of orthogonally polarized beams and introduces a further delay $\phi_{0}$ between each pair. When the four beams interfere at the sensor, $\gamma$ is encoded at the relative delay for each possible beam pair.

Figure 3(b) shows a test image measured using the multidelay configuration described, observing the same Cd lamp line as in Section II A. The combination of PPM and LPM terms can be seen in the zoomed view of the image, shown in Figure 3(c). Two Savart plates were available for use in this work, with thicknesses $4 \mathrm{~mm}$ and $2.2 \mathrm{~mm}$. Both plates are $\alpha$ BBO. For all multi-delay results shown here, these plates are aligned so as to function as a single Savart plate of thickness $L_{\mathrm{SP}}=6.2 \mathrm{~mm}$. For the system described, this gives a fringe period of $\sim 55$ pixels at $\lambda=434 \mathrm{~nm}-$ a sub-optimal spatial resolution. The waveplates in the $\psi_{0}$ and $\phi_{0}$ positions in Figure 3(a) have $L_{\mathrm{WP}}=4.48 \mathrm{~mm}$ and $L_{\mathrm{WP}}=6.35 \mathrm{~mm}$ respectively. In Figure 3(c) four interleaved images corresponding to the four polarizer orientations are shown separately.

\section{Demodulation}

Demodulation of the multi-delay interferogram to obtain $\zeta$ and $\Phi$ images for each of the four delays is more involved than in the single-delay case, requiring Fourier techniques. First, it is noted that the PPM has its spatial carrier frequencies at the $x$ and $y$ Nyquist frequencies $(0.5$ cycles/pixel). This can be seen in Figure 4(a), which shows the power spectral density (PSD) of the multi-delay test image from Figure 3(b). The (positive frequency) interference terms are labeled according to their imparted delay, using the symbols from Equation 10. The pure LPM term $\gamma\left(\psi_{0}\right)$ is sufficiently well separated in frequency space from the other terms that $\zeta\left(\psi_{0}\right)$ and $\Phi\left(\psi_{0}\right)$ images can be extracted using standard Fourierdomain filtering techniques ${ }^{36}$. The three remaining terms do not yield to Fourier-domain filtering in the same way due to aliasing. To demodulate these terms we use 'synchronous demodulation' 33 , which involves multiplying the interferogram by a reference image that is the PPM in complex exponential form: $\exp \left(\operatorname{im} \frac{\pi}{2}\right)$. Figure 4(b) shows the PSD of this 'synchronous product' for the same test image. By the Fourier transform shifting property, the $\gamma\left(\phi_{0}+m \frac{\pi}{2}\right)$ term is now shifted in frequency space from the $x$ and $y$ Nyquist frequencies down to zero. Similarly, the $\gamma\left(\phi_{0} \pm \psi_{0}+m \frac{\pi}{2}\right)$ terms are now shifted to the $\pm \psi_{0}$ spatial frequencies. All three remaining terms can now be demodulated using Fourier-domain

\begin{tabular}{lcc}
\hline \hline & Single-delay & Multi-delay \\
\hline$L_{\mathrm{WP}}: \phi(\mathrm{mm})$ & 4.48 & 6.35 \\
$L_{\mathrm{WP}}: \psi(\mathrm{mm})$ & - & 4.48 \\
$L_{\mathrm{SP}}(\mathrm{mm})$ & - & $4+2.2$ \\
\hline$\phi_{0}\left(10^{3} \mathrm{rad}\right)$ & $8.19 \pm 0.06$ & $11.8 \pm 0.1$ \\
$\psi_{0}\left(10^{3} \mathrm{rad}\right)$ & - & $8.19 \pm 0.06$ \\
$\phi_{0}+\psi_{0}\left(10^{3} \mathrm{rad}\right)$ & - & $20.0 \pm 0.1$ \\
$\phi_{0}-\psi_{0}\left(10^{3} \mathrm{rad}\right)$ & - & $3.6 \pm 0.1$ \\
\hline \hline
\end{tabular}

TABLE I. Setup and calibration information for the two CI instrument configurations used in Section IV of this work. $L_{\mathrm{WP}}$ and $L_{\mathrm{SP}}$ are waveplate and Savart plate thicknesses, respectively. Interferometer delays correspond to normal ray incidence and wavelength $\lambda_{0}=434.0 \mathrm{~nm}$.

filtering to obtain the $\zeta$ and $\Phi$ images $^{36}$.

\section{Interferometer delay calibration values}

To interpret $\zeta$ and $\Phi$, the absolute interferometer delay(s) must be known at the wavelength being observed. This cannot be directly inferred from a single $\Phi$ image since $\Phi$ is wrapped in the interval $(-\pi, \pi] \mathrm{rad}$. Measurements of $\Phi$ at multiple wavelengths are required. For each of the waveplates used here, this calibration procedure has already been carried out using a tuneable laser ${ }^{9,10}$. The delay values used in this work are based on these previous measurements and are listed in Table I for the single-delay and multi-delay instrument configurations used. The quoted uncertainty on these values accounts for both measurement error and estimated uncertainty in the dispersion model used to convert to the relevant wavelength. Repeated measurements of these delay values in the intervening years since the work by Silburn et al. ${ }^{10}$ show variability equal to or smaller than the uncertainty values quoted in Table I. The uncertainty in these delay calibration values is not accounted for in any analysis presented in this work.

In the next section, we look at how CI can be used to measure plasma electron density $n_{\mathrm{e}}$.

\section{COHERENCE IMAGING MEASUREMENT OF ELECTRON DENSITY}

\section{A. Modeling Balmer series lineshapes}

Simulations predict ${ }^{37}$ that the MAST-U divertor will operate with densities up to $3 \times 10^{20} \mathrm{~m}^{-3}$ and temperatures in the range $0.5<T_{\mathrm{e}}<5 \mathrm{eV}$. In these conditions the hydrogen Balmer series lineshapes are determined by Stark broadening, Doppler broadening and Zeeman splitting ${ }^{15,17}$. Spectroscopic measurement of $n_{\mathrm{e}}$ via Stark broadening relies on the fact that the Stark contribution to the line width is roughly $\propto n_{\mathrm{e}}^{2 / 3}$. The line choice for this $n_{\mathrm{e}}$ measurement is a trade-off: the further up the Balmer series (i.e. the higher the principal quantum number $n_{q}$ of the initial atomic state), the more pronounced the Stark broadening but the lower the brightness of the line ${ }^{19}$. In conventional divertor spectroscopy one or more of the $n_{q} \geq 6$ Balmer lines are typically observed simul- 
(a)

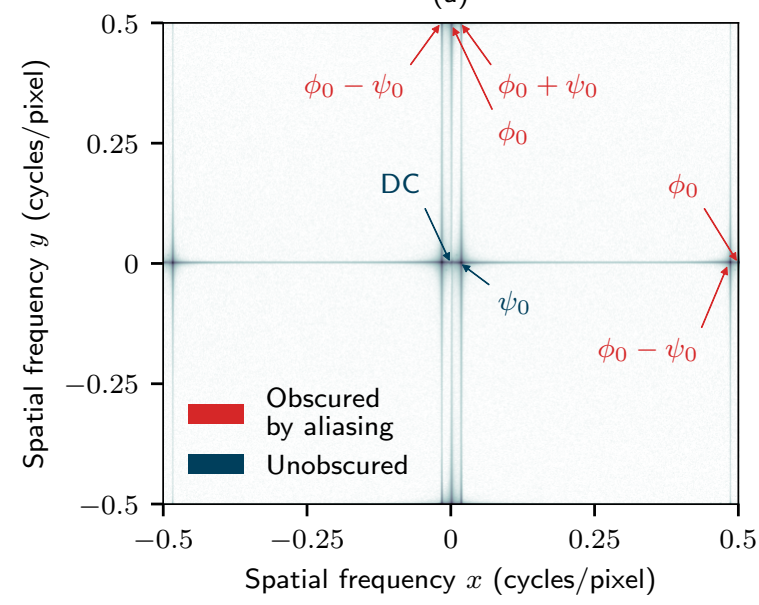

(b)

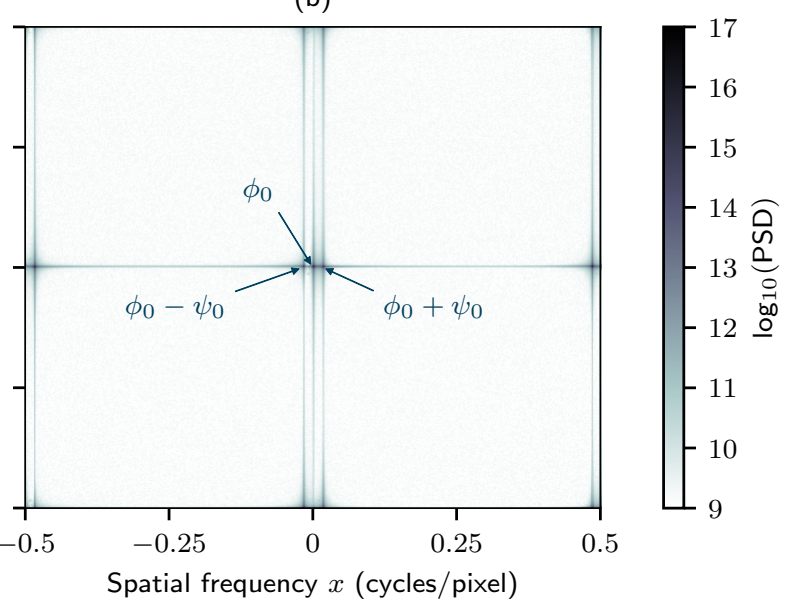

FIG. 4. (a) Power spectral density (PSD) estimate of the multi-delay test image plotted in Figure 3(b). (b) PSD estimate of the 'synchronous product' of the same image.

taneously for a robust $n_{\mathrm{e}}$ estimate ${ }^{15-18}$. However, in CI we are limited to observing only a single line, making the choice especially important. In this work, we consider the $\mathrm{H}_{\gamma}$ line $\left(n_{q}=5, \lambda_{0}=434.0 \mathrm{~nm}\right)$ only, as we will see that this gives good sensitivity across the relevant $n_{\mathrm{e}}$ range for the available CI interferometer delays.

In previous work ${ }^{20}$, the Stark-broadened $\mathrm{H}_{\gamma}$ lineshape was approximated using a Lorentzian function with width $\propto n_{\mathrm{e}}^{2 / 3}$. Here, we instead use a look-up table (LUT) of Stark-Zeeman lineshapes, generated for plasma diagnostics by Rosato et al. ${ }^{38}$ via numerical solution of the Schrödinger equation. This LUT spans an appropriate range of input values of $n_{\mathrm{e}}$, magnetic field strength $|\boldsymbol{B}|$ and electron temperature $T_{\mathrm{e}}$ - upon which the Stark profile has only a very weak dependence. The lineshape for an arbitrary set of input values can then be found via interpolation ${ }^{38}$. Since the relative strengths and polarization states of the observed Zeeman-split $\pi$ and $\sigma^{ \pm}$line components are determined by the angle between the magnetic field $\boldsymbol{B}$ and the line of sight ${ }^{39}$, the LUT contains lineshapes for both parallel $(\|)$ and orthogonal $(\perp)$ views. From these two views, the appropriate Stokes vector for the spectrum can be calculated for arbitrary view angles ${ }^{38,39}$. The Rosato et al. model assumes a pure deuterium plasma, but the result for pure hydrogen can be approximated by multiplying the input temperature by 2 (effectively dividing the ion mass by 2 in the calculation of ion thermal velocity) ${ }^{40}$.

For the Doppler broadening contribution to the lineshape we will, unless otherwise stated, use a simple Gaussian profile i.e. we will a Maxwellian velocity distribution for the emitting neutral species, with temperature $T_{\mathrm{n}}$. This assumption is justified in the context of the Magnum-PSI experiments in Section IV D. This Doppler-broadened profile is combined with the Stark-Zeeman profile of the Rosato et al. model by numerical convolution to give the final modeled lineshape.

Figure 5(a) uses this model to plot the $\mathrm{H}_{\gamma}$ lineshape for a range of $n_{\mathrm{e}}$, with $T_{\mathrm{e}}=T_{\mathrm{n}}=1 \mathrm{eV}$ and $|\boldsymbol{B}|=0 \mathrm{~T}$. The corresponding Lorentzian approximations (with the same Doppler contribution) are also plotted for comparison. For each of these lineshapes, Figure 5(b) plots the corresponding $\zeta$ profile as a function of interferometer delay, calculated using Equation 4. It is immediately clear that the Lorentzian approxima- tion can lead to a large underestimate of the $n_{\mathrm{e}}$ value inferred from $\zeta$ so it is not considered any further here. The four vertical dotted lines in Figure 5(b) correspond to the four delays of the multi-delay CI setup described in Section II B.

We will now consider in more detail the contribution of Zeeman splitting to the observed contrast. Since independent broadening / splitting contributions to the lineshape are combined by convolution ${ }^{19}$, the corresponding contributions to $\gamma$ (and so too to $\zeta$ ) are combined by multiplication (by the convolution theorem). For our purposes, Stark broadening and Zeeman splitting are independent effects. This can be verified by comparing the $\zeta$ profiles generated using the model described above for $|B|=1 \mathrm{~T}$ to those generated for $|\boldsymbol{B}|=0 \mathrm{~T}$ that have then been multiplied by $\zeta_{\mathrm{Z}}$, a Zeeman splitting contrast factor calculated using a simple 'strongfield' approximation ${ }^{4,39}$. The maximum absolute deviation between these two $\zeta$ profiles for the plasma parameters and delays considered in Figure 5 is 0.003 , which is smaller than we are able to measure here. In Figure 5(b), the black line indicates $\zeta_{Z}$ for $|\boldsymbol{B}|=1 \mathrm{~T}$ for a parallel view of the field. The pink shading then indicates the range of possible values that $\zeta_{z}$ can take for an orthogonal view of the field, depending on the relative orientation of the CI instrument's front polarizer and $\boldsymbol{B}$. This dependency arises as the $\sigma^{ \pm}$components are both linearly polarized in the direction of $\boldsymbol{B}$. It follows that, for an orthogonal view, a polarizer with the appropriate orientation can be used to suppress both $\sigma^{ \pm}$components, leaving only the central $\pi$ component and therefore setting $\zeta_{\mathrm{Z}} \approx 1$. This technique is often used in spectroscopy when the $\boldsymbol{B}$-field geometry is simple and known ${ }^{15,41}$. Figure 5(b) shows that the $\zeta_{\mathrm{Z}}$ contribution can be significant for low $n_{\mathrm{e}}$ and large delays, and also that the orientation of the front polarizer should be optimized and/or considered in the interpretation of results.

The $\zeta$ profile alone is enough to unambiguously reconstruct the lineshape only if the spectrum $g(v)$ is symmetric about $v_{0}$, its centre-of-mass frequency. This well-known result follows from Equation 4. Without this symmetry, the $\Phi$ profile is also required for the reconstruction. For a homogeneous emitter, the lineshape model described above is symmetric about $v_{0}$, and the analysis methods introduced in the next section will consider $\zeta$ only. However, an observed $g(v)$ that is 
(a)

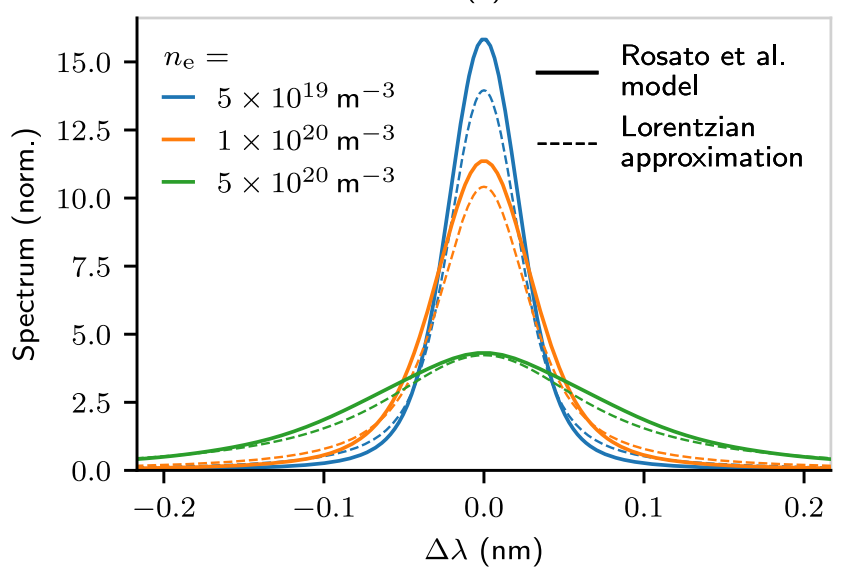

(b)

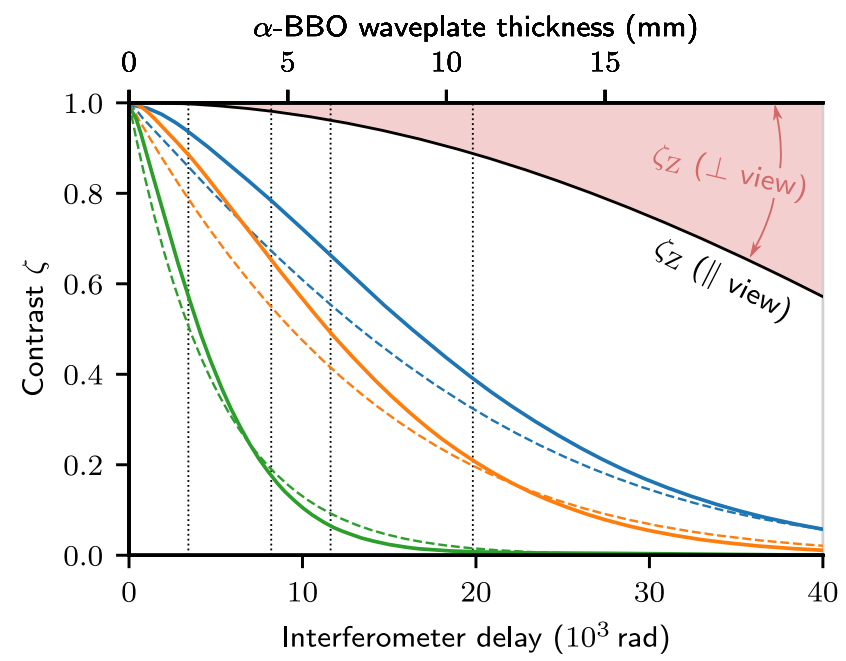

FIG. 5. (a) Modeled lineshapes for $\mathrm{H}_{\gamma}$ emission at $434.0 \mathrm{~nm}$ over a range of $n_{\mathrm{e}}$ for $T_{\mathrm{e}}=T_{\mathrm{n}}=1 \mathrm{eV}$ and $|\boldsymbol{B}|=0 \mathrm{~T}$. (b) The corresponding modeled $\zeta$ profiles as a function of interferometer delay.

line-integrated through an inhomogeneous emitter will not, in general, be symmetric due to Doppler shifts. Interpretation of line-integrated results in the context of Magnum-PSI will be discussed in Section IV D.

\section{B. Fitting to measured $\mathrm{Cl}$ contrast}

Inferring $n_{\mathrm{e}}$ from measurements of $\zeta$ made at one or more delays is a curve-fitting problem, requiring fast evaluation of the modeled $\zeta$. To do this we pre-calculated a $\zeta$ LUT on a grid spanning the expected range of $n_{\mathrm{e}}, T_{\mathrm{e}}=T_{\mathrm{n}}, \boldsymbol{B}$ and interferometer delay. LUT interpolation then allows for fast comparison to the data for fit optimization. For the curve-fitting framework, we use Bayesian parameter estimation. When fitting, we will assume that $\boldsymbol{B}$ and interferometer delay is known perfectly well and also that $T_{\mathrm{e}}=T_{\mathrm{n}}$. This leaves $n_{\mathrm{e}}$ and $T_{\mathrm{n}}$ as the free lineshape model parameters to be optimized.

For a single measurement of $\zeta$, the most that can be inferred about $n_{\mathrm{e}}$ without making assumptions about $T_{\mathrm{n}}$ is an upper limit. While previous $\mathrm{CI}$ work ${ }^{20}$ overcame this by assuming Doppler broadening to be negligible, we will make the more conservative assumption of a $6 \mathrm{eV}$ (soft) upper limit on $T_{\mathrm{n}}$, using the prior probability density function (PDF):

$$
P\left(T_{\mathrm{n}}\right) \propto \frac{1}{1+\exp \left(k\left[T_{\mathrm{n}}-6 \mathrm{eV}\right]\right)},
$$

where we choose $k=4 \mathrm{eV}^{-1}$. This PDF is plotted in Figure 6(a). The prior is only necessary for the single-delay CI data. The likelihood function for each $\zeta$ data point is assumed to be a normal distribution and noise on the measurements made at different delays and at different points in the image is assumed to be uncorrelated. The product of the prior PDF and total likelihood function at each image point is proportional to the posterior PDF, which can be evaluated on the LUT parameter grid to find the maximum a posteriori probability (MAP) estimate for parameters $n_{\mathrm{e}}$ and $T_{\mathrm{n}}$. Marginal posterior PDFs for $n_{\mathrm{e}}$ and $T_{\mathrm{n}}$ and confidence intervals are calculated using a Markov chain Monte Carlo (MCMC) method ${ }^{42}$.

In testing this LUT-based $\zeta$-fitting tool on modeled spectra, we can quantify the systematic error due to Doppler broadening - particularly important in the case of single-delay CI. To do this, $\zeta$ profiles are generated over a range of $n_{\mathrm{e}}$ and $T_{\mathrm{e}}=T_{\mathrm{n}}$. Zero-mean Gaussian noise is added to the modeled $\zeta$, with realistic standard deviation $\sigma_{\zeta}=0.02$. Then, $\zeta$ is fit to and the $n_{\mathrm{e}}$ MAP determined. For each set of inputs, the average $n_{\mathrm{e}}$ MAP is taken over 100 independent instances of the measurement noise. This procedure is carried out first for the single-delay CI configuration from Table I, and the results are plotted in Figure 6(a). This shows that the overestimate in $n_{\mathrm{e}}$ can be significant even for moderate $T_{\mathrm{n}}-$ e.g. $>25 \%$ at $n_{\mathrm{e}}=7 \times 10^{19} \mathrm{~m}^{-3}$ and $T_{\mathrm{n}}=3 \mathrm{eV}$. Figure $6(\mathrm{~b})$ then plots the same test, but for the multi-delay CI configuration from Table I. This shows a significant reduction in systematic error, extending the range over which $n_{\mathrm{e}}$ can be inferred with accuracy down to lower $n_{\mathrm{e}}$ and/or higher $T_{\mathrm{n}}$ conditions.

In the next Section, these methods are tested experimentally.

\section{EXPERIMENTAL TESTING ON MAGNUM-PSI}

\section{A. Setup and operation}

Magnum-PSI is a linear plasma experiment capable of producing steady-state plasma beams with temperatures $(0.1-5 \mathrm{eV})$, densities $\left(10^{19}-10^{21} \mathrm{~m}^{-3}\right)$ and field strengths $(0-2.5 \mathrm{~T})$, matching the divertor conditions of current and future tokamaks ${ }^{21}$. A cascaded arc source produces a plasma beam of diameter $\sim 20 \mathrm{~mm}$ that is confined by the field of five superconducting solenoid magnets. A movable, replaceable target sits $1.5 \mathrm{~m}$ downstream from the source, within a vacuum vessel of diameter $0.5 \mathrm{~m}$. The control parameters for Magnum-PSI are the source gas flow $Q_{\mathrm{S}}$ in standard liters per minute (SLM), source current $I_{\mathrm{S}}$ and $|\boldsymbol{B}|$. In this section, we use the CI techniques described above to measure the $n_{\mathrm{e}}$ profile of the Magnum-PSI beam for a range of plasma conditions, benchmarking our results against the Thomson scattering (TS) diagnostic system ${ }^{43}$, whose reported $n_{\mathrm{e}}$ measurement accuracy is better than 5\%. The measurements presented in this section were taken in parallel with a separate investigation into the performance of the MAST-U Langmuir probes ${ }^{44}$. 
(a)

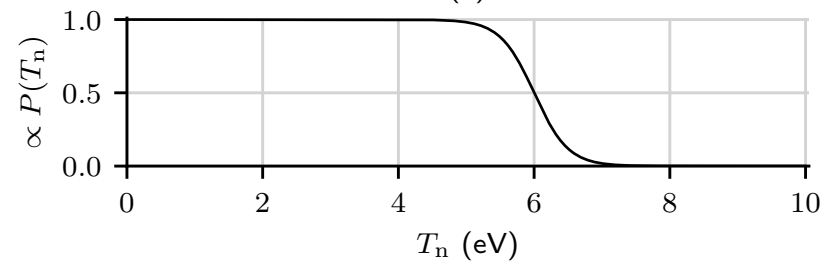

(b)

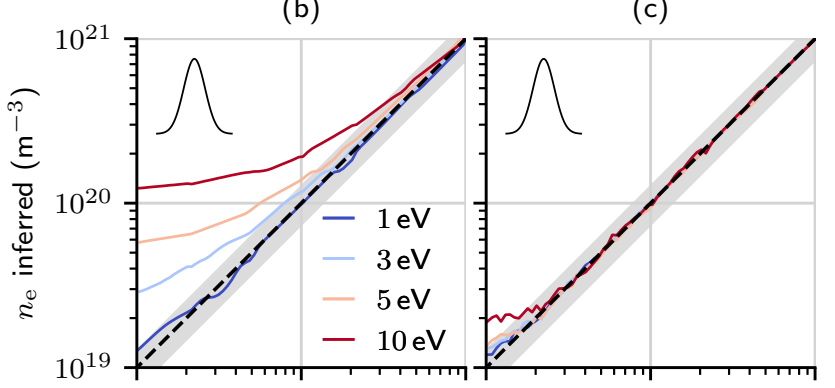

(d)

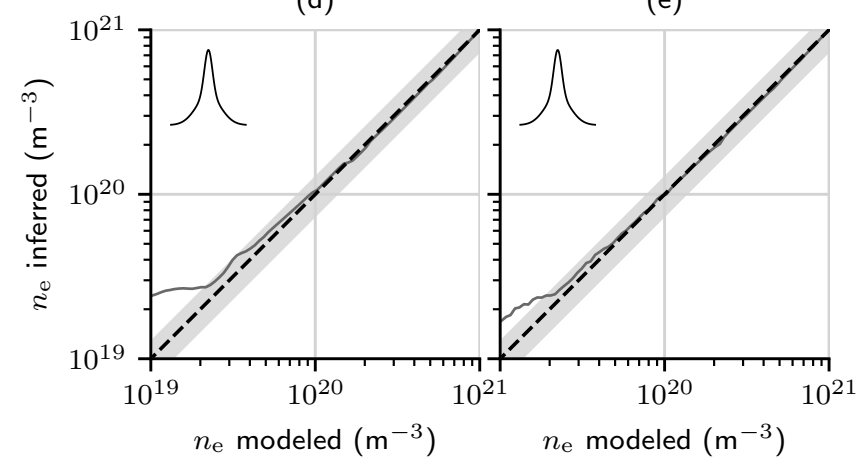

FIG. 6. (a) The prior PDF used when analysing single-delay CI data. (b) - (e) Predicted error in inferred $n_{\mathrm{e}}$ due to Doppler broadening as a function of modeled $n_{\mathrm{e}}$, where the gray shaded region indicates $\pm 25 \%$ error. (a) and (b): Gaussian Doppler broadening plotted across a range of $T_{\mathrm{n}}$ for (a) the single-delay CI configuration and (b) the multi-delay configuration (see Table I). (c) and (d): non-Gaussian Doppler broadening caused by two emitting hydrogen populations with different temperatures $(3 \mathrm{eV}$ and $0.3 \mathrm{eV})$ and intensity ratio 2:1. Shown for (c) single-delay and (d) multi-delay CI. The Doppler-broadened lineshape contribution is plotted inset for (b) $-(e)$.

The CI instrument was mounted on the railing of a mezzanine area, such that it's front pupil was a distance of $5.5 \mathrm{~m}$ from the Magnum-PSI beam, which it viewed through a $70 \mathrm{~mm}$ diameter port in the vacuum vessel. Usually this mount is occupied by a high-speed camera, far enough from the coils that the stray field does not affect operation. Figure 7(a) shows a photo of the installed CI setup in relation to the experiment. A telephoto DSLR lens with focal length $600 \mathrm{~mm}$ and $f / 6.3$ occupies the $l_{1}$ position in the CI instrument (see Figure 2(a)), while the $l_{2}$ and $l_{3}$ lenses have focal lengths $105 \mathrm{~mm}$ and $150 \mathrm{~mm}$ respectively. This provides a vertical field of view of $0.5^{\circ}$. The port views the beam a few $\mathrm{cm}$ upstream of the target, in the same plane as the vertical TS laser path and also the sight lines of a grating spectrometer. Figure 7(b) shows this geometry as viewed from the source. Figure 7(c) shows the measured transmission profile of the two-cavity interference filter used in the CI system to isolate the $\mathrm{H}_{\gamma}$ emission. Also shown is an example line spectrum measured by the spectrom- (a)
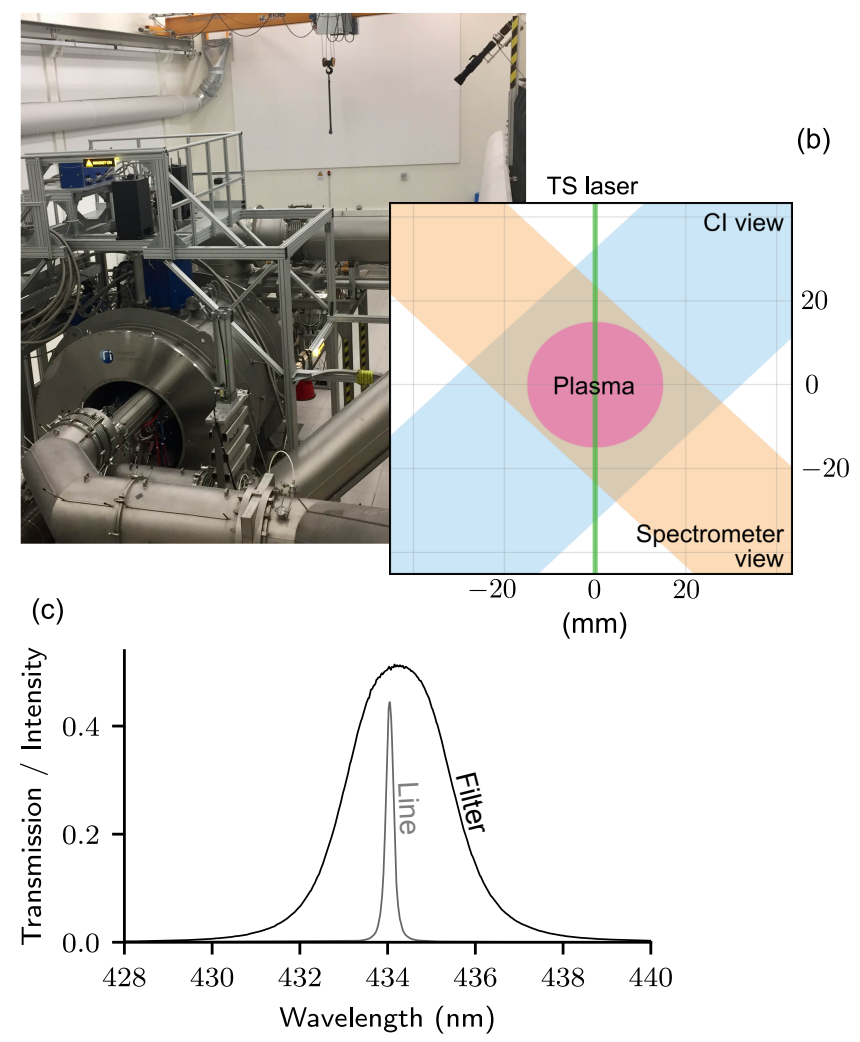

FIG. 7. (a) The Magnum-PSI experiment hall. The CI instrument can be seen in the top-right corner. (b) The CI and spectrometer views relative to the TS laser path and the plasma beam, as viewed from the plasma source. (c) Measured transmission profile of the narrowband interference filter and $\mathrm{H}_{\gamma}$ spectrum observed by the spectrometer.

eter looking through the beam axis.

The CI camera was connected to a PC on the mezzanine and was remotely controlled from the experiment control room. Before the start of operations, the CI temperature controller was set to $35 \pm 0.25^{\circ} \mathrm{C}$ and left for more than 2 hours to equilibrate. Figure 9(a) shows an example interferogram for the multi-delay system viewing the Magnum-PSI beam. This image was exposed for $t_{\exp }=0.8 \mathrm{~s}$ and the plasma control parameters are given in the figure caption. At this display resolution, the pixelated pattern is not visible. The peak $n_{\mathrm{e}}$ and $T_{\mathrm{e}}$ on-axis reported by the TS diagnostic for this discharge is $n_{\mathrm{e}}=7.90 \times 10^{20} \mathrm{~m}^{-3}$ and $T_{\mathrm{e}}=1.77 \mathrm{eV}$. Figure $9(\mathrm{~b})$ shows the extracted brightness image.

\section{B. Calibration}

In CI, a multiplicative contrast degradation factor $\zeta_{\mathrm{I}}$ determines the maximum observable fringe contrast - analogous to the instrument function of a grating spectrometer ${ }^{3}$. Here, $\zeta_{\mathrm{I}}$ was measured using a Cd lamp illuminating an integrating sphere, roughly every two hours during operations. The instrument was unmounted and calibration images were captured of the $\mathrm{Cd}$ I line at $467.8 \mathrm{~nm}$, the appropriate bandpass filter having been swapped in. Figure 8 plots the multi-delay $\zeta_{\mathrm{I}}$ value for each of the four delays, averaged over a central $20 \times 20$ pixel region. The values shown are averaged over 


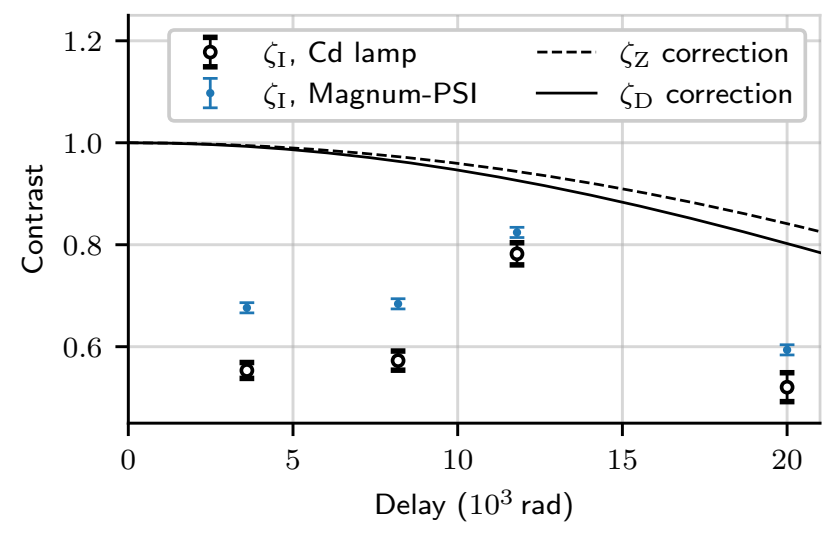

FIG. 8. Measured instrument contrast calibration values for multidelay $\mathrm{CI}$.

4 measurements taken over the course of the day, with error bars indicating the corresponding standard deviation. The $\zeta_{\mathrm{I}}$ images were uniform to within \pm 0.02 . It is worth noting first that $\zeta_{\mathrm{I}}$ for the pure PPM term (at $\phi_{0} \approx 12000$ rad delay) is significantly higher than for the LPM terms. One possible cause for this is that only a single crystal plate encodes this delay, since variations in the thickness of each plate contribute to the range of phase delays imparted across the plate aperture, reducing $\zeta_{\mathrm{I}}$.

It was later found that, for the multi-delay system, a higher $\zeta$ was observed during Magnum-PSI discharges than for the Cd lamp measurements of $\zeta_{\mathrm{I}}$ — indicating a systematic error in those measurements. Figure 8 also plots the multi-delay $\zeta_{I}$ values estimated from Magnum-PSI measurements made on the same day. Specifically, these values are from the far edges of the Magnum-PSI beam (impact parameter $|b|>15 \mathrm{~mm}$ ), and from the discharges with the lowest TS-reported $n_{\mathrm{e}}$. Two correction factors have been divided out to obtain these values: $\zeta_{\mathrm{Z}}$ to account for Zeeman splitting at $|\boldsymbol{B}|=1.2 \mathrm{~T}$ (the dashed line) and $\zeta_{\mathrm{D}}$ to account for Doppler broadening at $T_{\mathrm{n}}=0.75 \mathrm{eV}$ (the solid line). This $\zeta_{\mathrm{D}}$ profile was chosen based on the corresponding spectrometer measurement at the same line-of-sight impact parameter $b$, meaning these $\zeta_{\mathrm{I}} \mathrm{CI}$ calibration values are partially derived from the instrument function calibration of the spectrometer.

The cause of the discrepancy between these two sets of $\zeta_{\mathrm{I}}$ measurements is not fully understood. Subsequent lab testing rules out the wavelength dependence of $\zeta_{\mathrm{I}}$ as a significant contribution (the $\mathrm{Cd} \mathrm{I}$ line is over $30 \mathrm{~nm}$ from $\mathrm{H}_{\gamma}$ ). Testing also suggests that the cause could have been movement of the interferometer crystals during the repeated unmounting and re-mounting of the instrument for the $\mathrm{Cd}$ calibration measurements. For the calibration of all the the multi-delay data shown in this work, we use the $\zeta_{\mathrm{I}}$ values inferred from the Magnum-PSI measurements and spectrometer instead of the Cd lamp measurements. It should be noted that the singledelay $\zeta_{\mathrm{I}}$ calibration values were consistent between the $\mathrm{Cd}$ lamp measurements and the values estimated using method above. The design and calibration of the MAST-U CI instruments are being improved and will be discussed in a future publication.

Dark frames were also captured with each $\zeta_{\mathrm{I}}$, and were subtracted from all images before demodulation. We will refer to the line-integrated contrast extracted from the raw measurements of Magnum-PSI as $\breve{\zeta}$ so as to distinguish it from the Abel-inverted local contrast profiles $\zeta$ introduced in Section IV E. Each $\breve{\zeta}$ image was divided through by the corresponding $\zeta_{\mathrm{I}}$ image prior to analysis. Figure 9(c) shows the calibrated $\breve{\zeta}$ images for the four delays of the multi-delay data, in ascending order. In Figure $9(\mathrm{~d}), \breve{\zeta}$ is plotted as a function of delay for the central column of these images for two impact parameters. Also plotted are the corresponding $\breve{\zeta}$ profiles as predicted by the grating spectrometer measurements of the lineshape taken during the same discharge (instrument function deconvolved). The two instruments are in broad agreement.

\section{Continuum emission}

Even with the filter in place, it was found that the continuum emission reduced the measured contrast appreciably for some discharges. For the single-delay measurements, this cannot be accounted for a priori, while for the multi-delay measurements it is accounted for with a third fit parameter. If the (area-normalized) observed spectrum is the sum of line (L) and continuum (c) components, we have

$$
g(v)=I_{\mathrm{L}} g_{\mathrm{L}}(v)+I_{\mathrm{c}} g_{\mathrm{c}}(v),
$$

where the relative intensities satisfy $I_{\mathrm{L}}+I_{\mathrm{c}}=1$. Using Equation 4 , we can write the observed coherence as

$$
\gamma=\gamma_{\mathrm{L}}\left(I_{\mathrm{L}}+I_{\mathrm{c}} \frac{\gamma_{\mathrm{c}}}{\gamma_{\mathrm{L}}}\right),
$$

From the Fourier transform magnitude of the filter transmission bandpass (the dashed line in Figure 9(d)), it is clear that for all four of the delays used in this work, we have $\left|\gamma_{\mathrm{c}}\right| \approx 0$. It follows that the observed contrast is $\zeta \equiv|\gamma| \approx I_{\mathrm{L}}\left|\gamma_{\mathrm{L}}\right|$, i.e. the continuum manifests as a multiplicative contrast degradation factor, independent of delay, that indicates the fraction of line emission.

Since the line-integrated profiles do not show significant variation along the beam axis, for the remainder of this work we will present results from a central column slice through the images only.

\section{Non-Gaussian Doppler broadening and Doppler shifts}

The presence of non-Gaussian Doppler broadening or significant Doppler shifts could complicate the interpretation of the CI data. Previous work by Shumack et al. ${ }^{41}$ used a grating spectrometer to view $\mathrm{H}_{\beta}(486.1 \mathrm{~nm})$ at the plasma source of the Pilot-PSI machine (a smaller, non-superconducting forerunner to Magnum-PSI that used the same cascaded arc plasma source). From the observed lineshape, the presence of two atomic hydrogen populations was inferred: one coupled to the ions via charge exchange with $T_{\mathrm{n}}$ at a few eV and the other one cold at $0.1-0.5 \mathrm{eV}$. The intensity ratio between the two populations was constant across the beam profile at roughly 2 to 1 (hot to cold). In addition, the hot population was observed to be rotating around the beam axis, at velocities up to $10 \mathrm{~km} / \mathrm{s}$, due to an $\boldsymbol{E} \times \boldsymbol{B}$ drift. Since this rotation was observed to decrease with axial distance from the source, 
(a)

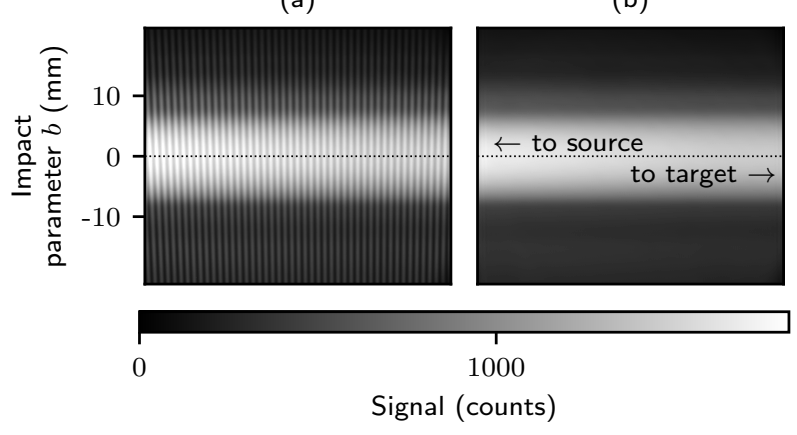

(c)

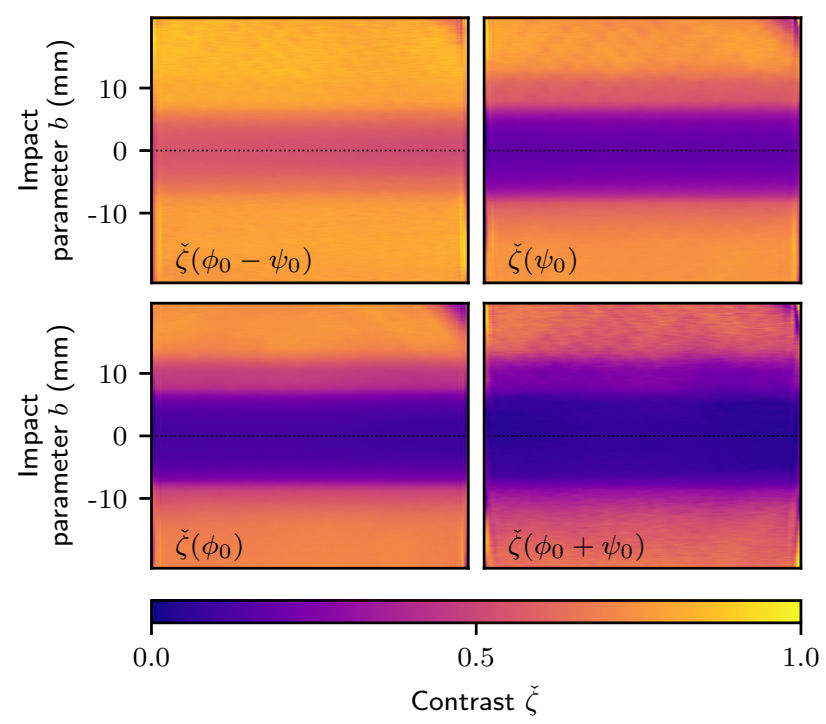

(d)

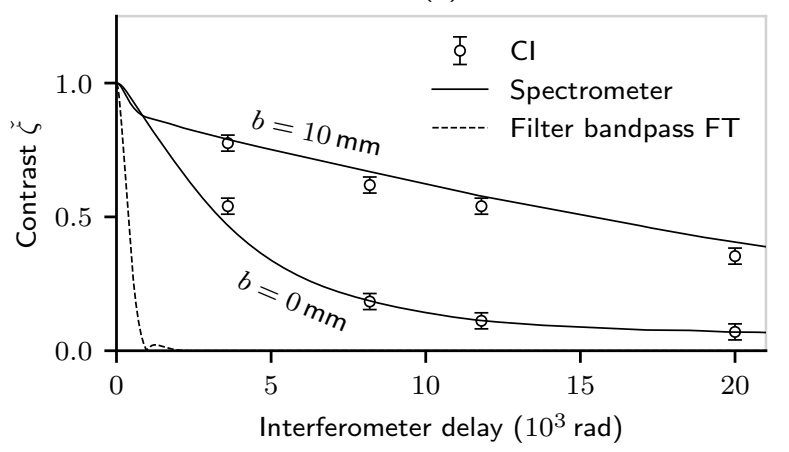

FIG. 9. (a) The raw $\mathrm{CI}$ interferogram observing $\mathrm{H}_{\gamma}$ emission from Magnum-PSI, with $I_{s}=200 \mathrm{~A},|\boldsymbol{B}|=1.2 \mathrm{~T}$. Instrument is in the multi-delay configuration and exposure time is $0.8 \mathrm{~s}$. (b) The brightness profile extracted from (a). (c) The contrast $\breve{\zeta}$ (line-integrated and calibrated) extracted from (a). (d) $\breve{\zeta}$ at the image center column plotted as a function of delay for two different view impact parameters. Plotted for comparison is the corresponding $\breve{\zeta}$ calculated from spectrometer measurements, with view orthogonal to CI.

with a characteristic decay length of $0.5 \mathrm{~m}$, it is not observed in our measurements at the Magnum-PSI target, which sits $1.5 \mathrm{~m}$ from the source.

The presence of two emitting populations at different temperatures results in a non-Gaussian Doppler broadening contribution to the lineshape, which could introduce systematic error into the inferred $n_{\mathrm{e}}$ if not accounted for. The size of this error was modeled by the same fitting procedure used in Section III B. Representative $T_{\mathrm{n}}$ chosen for the hot and cold populations in this test are $3 \mathrm{eV}$ and $0.3 \mathrm{eV}$. Figure 6(c) shows the predicted error for the single-delay CI configuration and Figure 6(d) shows the error for the multi-delay CI configuration. As might be expected, the presence of the cold population reduces the error due to Doppler broadening in the single-delay case when compared to the Gaussian broadening model with $T_{\mathrm{n}}=3 \mathrm{eV}$. In the case of the multi-delay CI, the error in inferred $n_{\mathrm{e}}$ is only larger than $5 \%$ for $n_{\mathrm{e}}<5 \times 10^{19} \mathrm{~m}^{-3}$. Since the predicted effect is small, we do not consider non-Gaussian Doppler broadening in the analysis of the Magnum-PSI CI results.

\section{E. Abel inversion}

The spectrum observed at each pixel has been integrated along a path $L$ through the plasma. It can be shown that ${ }^{5}$, provided the range of Doppler shifts along $L$ is small, the observed contrast $\breve{\zeta}\left(\phi_{0}\right)$ is related to the local contrast $\zeta\left(\phi_{0}, \boldsymbol{r}\right)$ at point $\boldsymbol{r}$ by:

$$
\check{\zeta}\left(\phi_{0}\right) \approx \frac{1}{I} \int_{L} \varepsilon(\boldsymbol{r}) \zeta\left(\phi_{0}, \boldsymbol{r}\right) d l .
$$

Here, $\varepsilon(\boldsymbol{r})$ is the local emissivity, satisfying

$$
I=\int_{L} \varepsilon(\boldsymbol{r}) d l
$$

Since $\check{\zeta}$ is weighted by $\varepsilon(\boldsymbol{r})$, Equation 15 must be inverted to yield $\varepsilon(\boldsymbol{r})$ before Equation 14 can be inverted to yield $\zeta\left(\phi_{0}, \boldsymbol{r}\right)$, from which $n_{\mathrm{e}}$ can be determined according to Section III B.

The Magnum-PSI plasma beam has approximate cylindrical symmetry about its axis ${ }^{45}$. This cylindrical symmetry is often assumed during spectroscopic studies of MagnumPSI (and also of Pilot-PSI), with an Abel inversion used to obtain the local plasma properties from the observed lineintegrals ${ }^{20,41,46}$. In this work, we will make the same assumption. Recovering the full profile of a non-symmetric beam requires multiple views from different directions, but these are not available for CI at this time. Since the CI and spectrometer have near-orthogonal views of the beam, the data from both diagnostics could feasibly be combined to perform a full tomographic inversion of the profile, but this is not attempted here.

Equations 14 and 15 are Abel-inverted using the simultaneous algebraic reconstruction technique (SART) ${ }^{47}$, an iterative technique that has been used in previous CI work ${ }^{9,10}$. Each pixel's line of sight is assumed to be narrow and to have equal collection power along its length. Line-integrated brightness profiles are brought smoothly to zero outside of the image edges prior to inversion. The position of the beam axis on the line-integrated profiles is estimated by choosing the brightest point in the smoothed, line-integrated brightness profile (See the horizontal black dotted lines in Figure 9). The radial inversion grid chosen extends to $r=30 \mathrm{~mm}$ and has 300 bins, for a grid spacing of $0.1 \mathrm{~mm}$ in this direction. For the multi-delay CI results, the $\breve{\zeta}$ profile is inverted independently for each of the four delays. 


\section{F. Comparison with Thomson Scattering}

Once the radial $\zeta$ profile(s) have been calculated, the fitting procedure described in III B is used to infer the corresponding profile for $n_{\mathrm{e}}$. Figures $10(\mathrm{a})-(\mathrm{d})$ plot the multi-delay CI results for an ascending scan in beam $n_{\mathrm{e}}$. See the figure caption for the corresponding Magnum-PSI control parameters. The data in Figure 10(d) corresponds to the pre-inversion images shown in Figure 9. The top row of Figure 10 shows 3-D plots of the inverted $\zeta$ profiles in black as a function of beam radius and delay. The grey mesh surface represents the MAP values for the fit to the $\zeta$ data at each $r$, plotted over a range of delays. Three slices through this surface are made at radial positions $r=0,6$ and $12 \mathrm{~mm}$, plotted in blue, orange and green respectively. In the second row, each of these slices is plotted as a function of delay only, along with the corresponding four $\zeta$ data points used to constrain the fit, allowing for visual inspection. The third row of the figure then plots the radial $n_{\mathrm{e}}$ profiles as inferred from both the CI and the TS (upper and lower halves of the profile). For the CI results, the MAP values are plotted as a solid line and the $68 \%$ confidence interval is shaded in gray. The $r$ values of the three slices are indicated with vertical lines of the appropriate color. Finally, the fourth row plots the corresponding radial temperature profiles: $T_{\mathrm{e}}$ for the TS and $T_{\mathrm{n}}$ for CI. For the CI data, only the $68 \%$ confidence interval is shown.

The agreement between the inverted $\zeta$ data and the fit profiles is good for these measurements, particularly closer to the beam axis. Agreement between CI and TS $n_{\mathrm{e}}$ profiles is good across the range shown. The peak $n_{\mathrm{e}}$ for the discharge shown in Figure $10(\mathrm{~d})$ is $n_{\mathrm{e}} \approx 8 \times 10^{20} \mathrm{~m}^{-3}$, high enough that the fringe contrast goes to zero for two (almost three) of the four interferometer delays. Despite this, good agreement with TS is maintained since Stark broadening is the dominant effect at high $n_{\mathrm{e}}$. At lower $n_{\mathrm{e}}$, the effect of the continuum emission, which can be inferred from the intercept of the fit curves with the contrast axis, can be significant. For the discharge shown in Figure 10(a), the fraction of the total observed emission that is attributed to continuum by the fit is $35 \%$ on-axis. This is consistent with the spectrometer data for the same discharge. For higher $n_{\mathrm{e}}$, there is negligible continuum emission on-axis and a slight contribution off-axis. A possible source of this continuum emission is the molecular hydrogen dissociative continuum.

The $T_{\mathrm{n}}$ inferred from the CI data is typically higher than the $T_{\mathrm{e}}$ reported by the TS, particularly for higher $n_{\mathrm{e}}$ discharges. This discrepancy is consistent with previous spectroscopic measurements of $T_{\mathrm{n}}$ on Pilot-PSI ${ }^{41}$.

Figure 11 plots inverted single-delay CI results from Magnum-PSI, presented in the same format as Figure 10. Results are taken from another low-to-high scan in $n_{\mathrm{e}}$, but this time at $|\boldsymbol{B}|=0.8 \mathrm{~T}$. See the figure caption for details of the Magnum-PSI control parameters. While the $n_{\mathrm{e}}$ profile inferred from single-delay CI shows good agreement with the TS for $n_{\mathrm{e}}>10^{20} \mathrm{~m}^{-3}$, Figure 11(a) shows that at lower densities $\left(n_{\mathrm{e}} \approx 5 \times 10^{19} \mathrm{~m}^{-3}\right)$ we see a considerable $(\sim 200 \%)$ overestimate in the $\mathrm{CI} n_{\mathrm{e}}$.

Finally, Figure 12 plots the CI $n_{\mathrm{e}}$ values on the plasma beam axis against the corresponding TS $n_{\mathrm{e}}$ values for multiple discharges measured with (a) the single-delay CI and (b) the multi-delay CI. The relative overestimate in $n_{\mathrm{e}}$ in the single- delay data is likely due to a combination of Doppler broadening and continuum emission. This highlights the difficulty in interpreting single-delay CI results in isolation.

\section{G. Discussion}

The measurement performance achieved with the tested instruments are now summarized. The time resolution was limited by the brightness of the observed emission. Since we were viewing a small plasma volume from far away, long exposure times in the range $0.5-10 \mathrm{~s}$ were needed to achieve a reasonable signal level, particularly at low $n_{\mathrm{e}}$. The approximate spatial resolution for the single-delay CI instrument, assuming detector-limited operation, was $0.04 \mathrm{~mm}$ for both image dimensions. For the multi-delay CI instrument, the approximate spatial resolution at the beam was $0.04 \mathrm{~mm}$ in the vertical direction and $2 \mathrm{~mm}$ in the horizontal direction. The horizontal resolution here was limited by the spatial frequency of the sinusoidal fringe pattern. Using a thicker Savart plate or a lens with a shorter focal length in the $l_{3}$ position would have given a higher horizontal spatial resolution, up to a practical limit of $0.25 \mathrm{~mm}$. The estimated $n_{\mathrm{e}}$ uncertainty achieved for the multi-delay CI was $40 \%$ for $n_{\mathrm{e}}=5 \times 10^{19} \mathrm{~m}^{-3}$ and $10 \%$ for $n_{\mathrm{e}}=5 \times 10^{20} \mathrm{~m}^{-3}$.

The performance of the technique will be different when it is applied to MAST-U (or to another tokamak). On MAST-U, the $\mathrm{H}_{\gamma}$ channel of the multi-wavelength imaging (MWI) diagnostic ${ }^{8}$, which looks tangentially into the divertor, achieves a reasonable signal level running at $200 \mathrm{~Hz}$ with an exposure time of $4.5 \mathrm{~ms}$. The average CI signal will be at least $50 \%$ lower than for the equivalent MWI filtered imaging channel (assuming a net unpolarized spectrum), but this performance can be taken as being indicative of the expected time resolution for the MWI's $\mathrm{H}_{\gamma}$ CI channel. The camera used in this work is limited to a framerate of $75 \mathrm{~Hz}$, so the limiting factor could instead be the choice of camera. The MWI has a spatial resolution of $5 \mathrm{~mm}$ for each of its channels, limited by optical aberrations ${ }^{8}$. The spatial resolution of the MWI CI system will depend on the CI configuration, and will be considered in a future publication.

\section{SUMMARY AND CONCLUSIONS}

In this work we have introduced new methods for coherence imaging, a narrowband spectral imaging technique for diagnosing fusion plasma experiments. This was motivated by wanting to maximize the resolution and coverage of measurements of the divertor plasma on the MAST-U spherical tokamak experiment. We applied the method of pixelated phase mask interferometry, already an established technique in optics, to CI, noting that it can achieve a higher (average) spatial resolution than the existing linear phase mask CI design. As well as a snapshot, single-delay PPM CI instrument, we introduced one example of a snapshot multi-delay instrument, suitable for measuring more complex spectra, that uses a combination of the PPM and LPM encodings.

We discussed in detail the application of CI to the measurement of electron density via Stark broadening of hydrogen Balmer line emission in plasma conditions relevant to the 

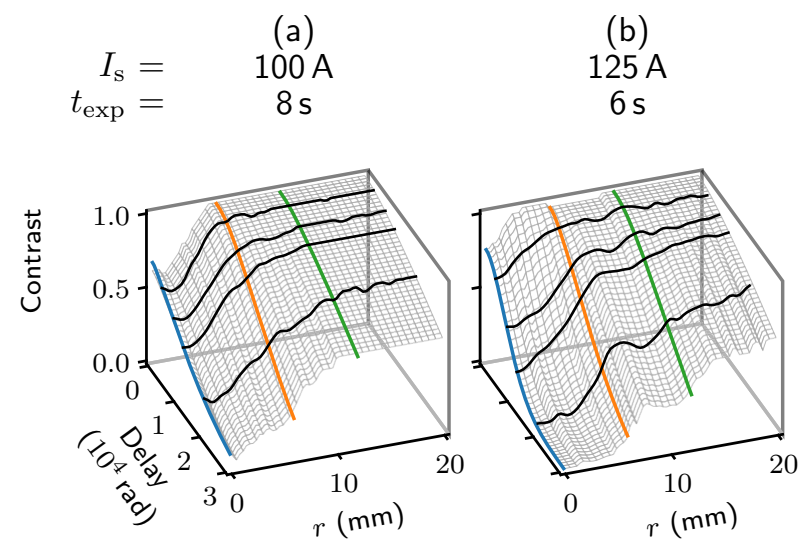

(b)

$125 \mathrm{~A}$
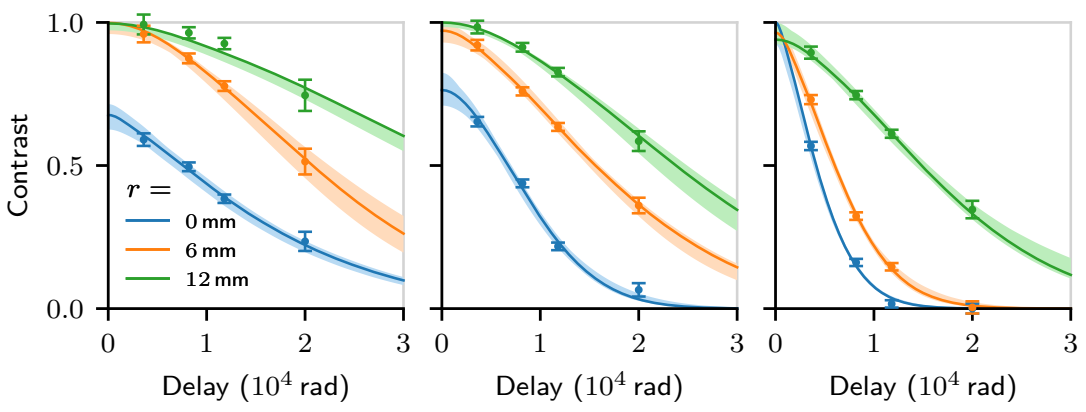

$160 \mathrm{~A}$

$1 \mathrm{~s}$
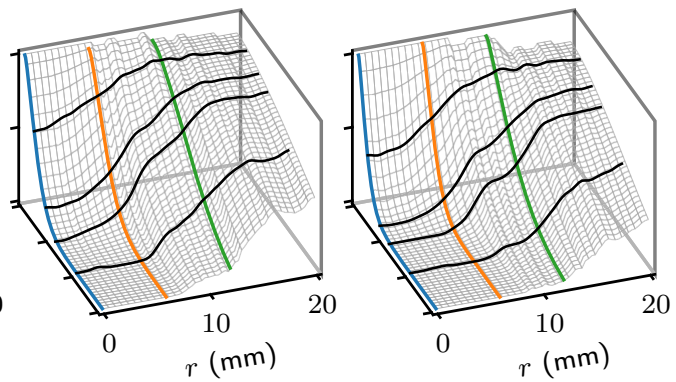

(d)

$200 \mathrm{~A}$

$0.8 \mathrm{~s}$
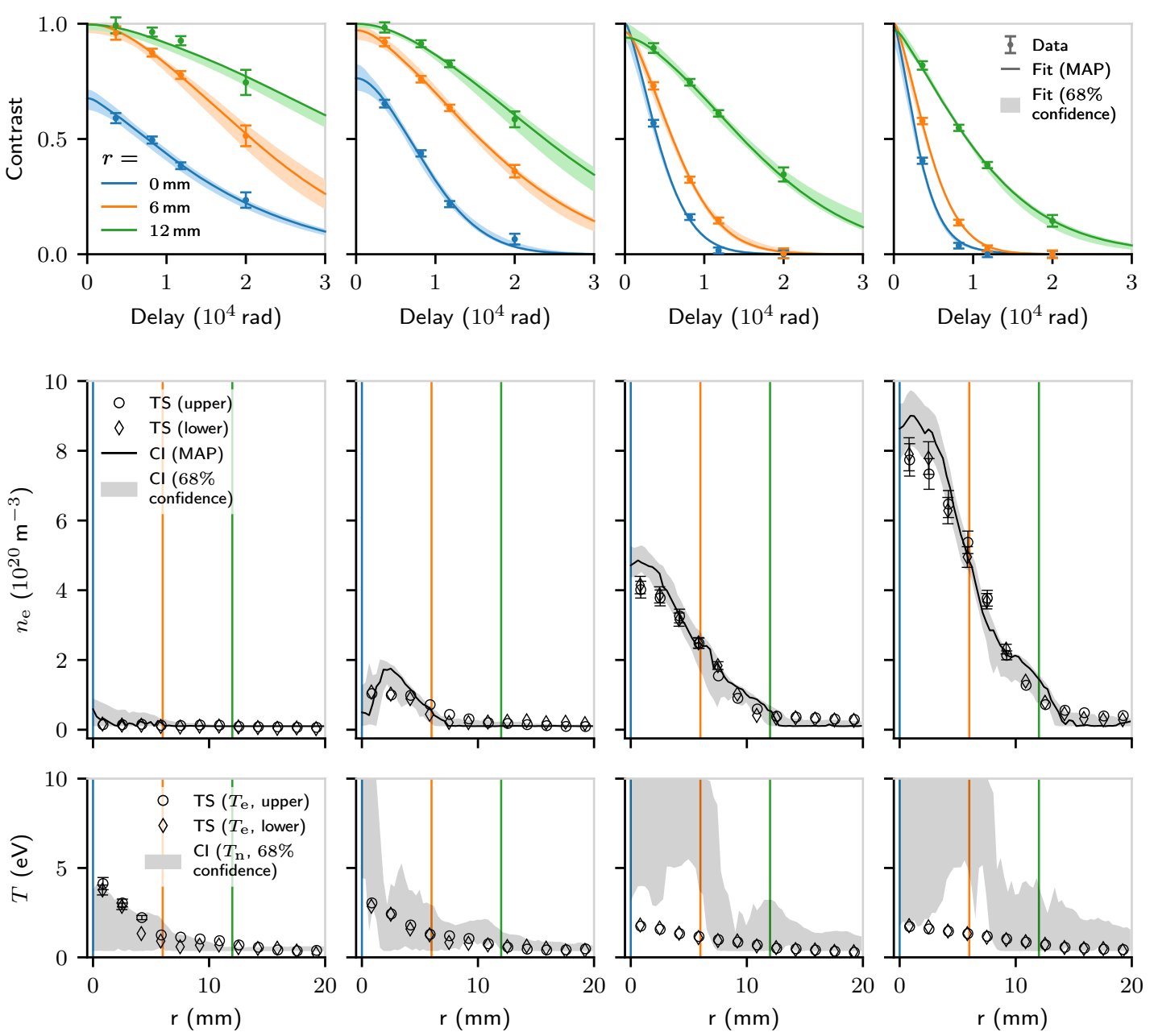

FIG. 10. Inverted CI results (multi-delay) where columns (a)-(d) correspond to a scan in Magnum-PSI source current $I_{\mathrm{s}}$. For these plasmas, source gas flow rate is $Q_{\mathrm{s}}=5 \mathrm{SLM}$ and $|\boldsymbol{B}|=1.2 \mathrm{~T}$. In the top row, inverted $\zeta$ profiles are plotted in black vs. delay and plasma beam radius $r$. The grey mesh surface is the best fit to this data. The second row plots slices at three different $r$, showing both data and fit. The third row then compares the $n_{\mathrm{e}}$ profiles inferred by CI and Thomson scattering (TS). The final row compares the neutral temperature $T_{\mathrm{n}}$ inferred from the CI data to the measured TS $T_{\mathrm{e}}$ profiles

study of tokamak divertor physics. The tabulated lineshape model of Rosato et al. ${ }^{38}$ was incorporated into an LUT-based fitting procedure for inferring $n_{\mathrm{e}}$ from measurements of CI fringe contrast. Tests of this fitting procedure using synthetic data showed that a multi-delay instrument configuration can significantly widen the dynamic range of the $n_{\mathrm{e}}$ measurement by improving robustness against Doppler broadening effects at lower $n_{\mathrm{e}}$.

Experimental CI measurements were made of divertorrelevant plasma conditions on the Magnum-PSI linear plasma experiment at DIFFER using the single-delay and multi-delay instrument configurations. For multi-delay CI measurements 

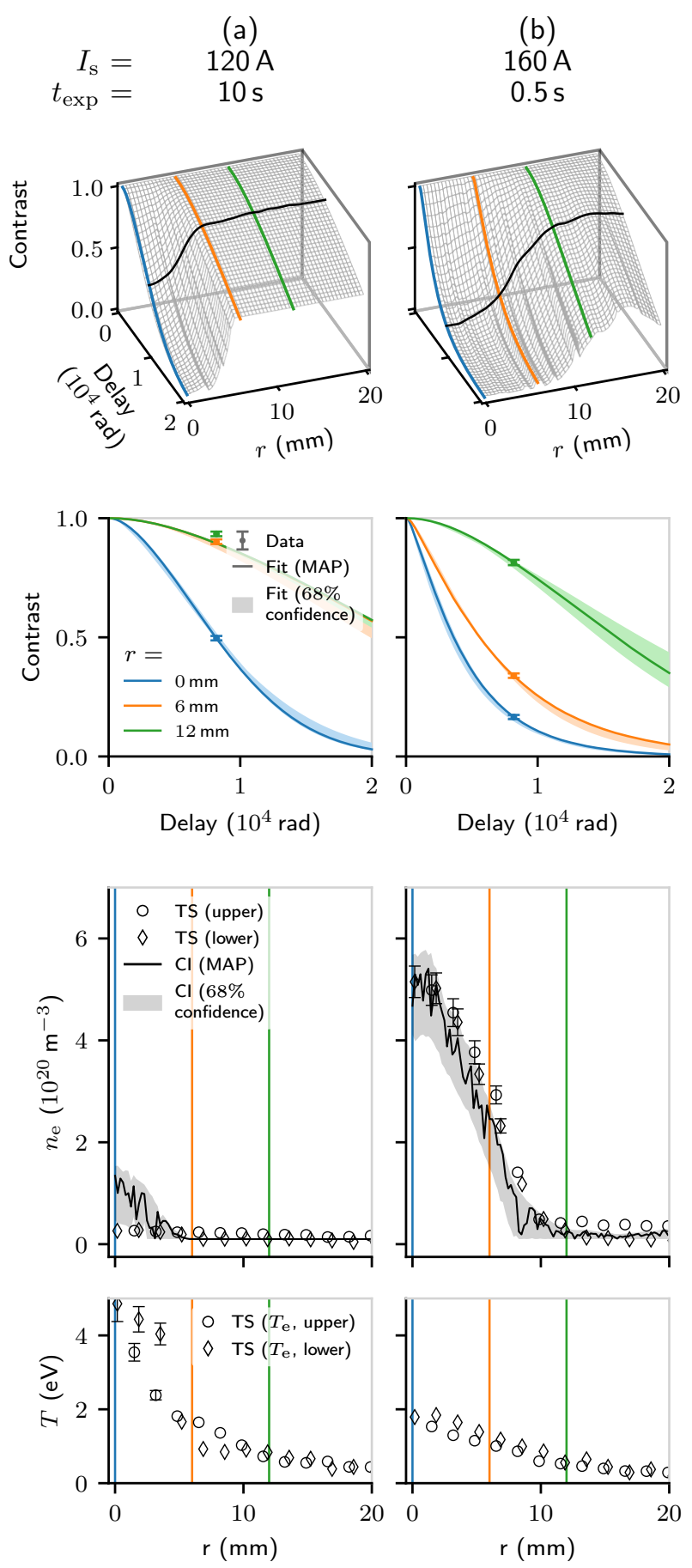

FIG. 11. Inverted CI results (single-delay) where columns (a)-(b) correspond to low and high Magnum-PSI source current $I_{\mathrm{s}}$. Source gas flow rate is $Q_{\mathrm{s}}=4.5 \mathrm{SLM}$ and $|\boldsymbol{B}|=0.8 \mathrm{~T}$ for both discharges shown. Results are presented in the same format as in Figure 10.

of $\mathrm{H}_{\gamma}$ emission, good agreement was found between the inferred $n_{\mathrm{e}}$ profiles and those measured using Thomson scattering across the range $3 \times 10^{19} \mathrm{~m}^{-3}<n_{\mathrm{e}}<1 \times 10^{21} \mathrm{~m}^{-3}$. For the single-delay CI measurements, good agreement with TS was only achieved for $n_{\mathrm{e}}>1 \times 10^{20} \mathrm{~m}^{-3}$, due to a combination of Doppler broadening and continuum emission. This highlights the difficulty of interpreting single-delay CI data in isolation when observing complicated spectra. (a)

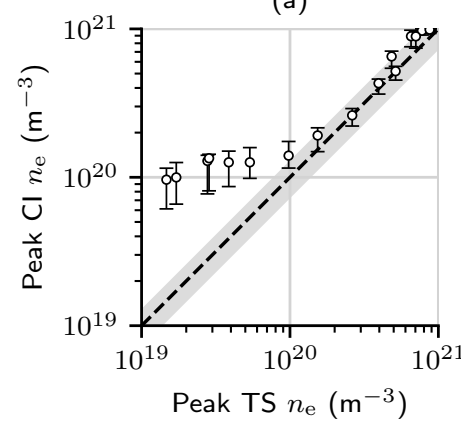

(b)

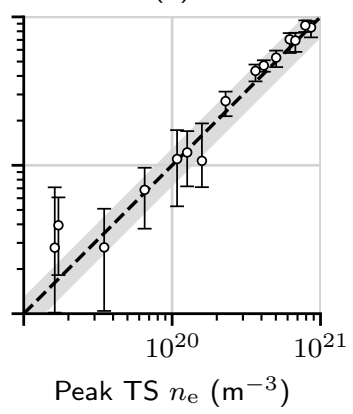

FIG. 12. Inferred $n_{\mathrm{e}}$ on the Magnum-PSI beam axis inferred using coherence imaging plotted against the corresponding $n_{\mathrm{e}}$ inferred using Thomson scattering for (a) the single delay CI and (b) multi-delay CI. Each data point corresponds to an independent Magnum-PSI discharge.

The techniques described in this work are currently being applied to MAST-U to help us better understand the physics of tokamak heat exhaust.

\section{ACKNOWLEDGMENTS}

This work has been carried out within the framework of the EUROfusion Consortium and has received funding from the Euratom research and training programme 2014-2018 and 2019-2020 under grant agreement No 633053. The views and opinions expressed herein do not necessarily reflect those of the European Commission. This work also received funding from the RCUK [grant numbers EP/T012250/1, EP/L01663X/1]. The authors would like to thank the team at Magnum-PSI for facilitating our experiments, in particular the help of Serje Brons, Thomas Morgan and Gijs Akkermans, Jack Leland and Fabio Federici. This work also benefited from conversations with Clive Michael, Chris Bowman, Sam Gibson, Kevin Verhaegh and Joël Rosato.

\section{DATA AVAILABILITY}

The data that support the findings of this study are available from the corresponding author upon reasonable request.

\section{Appendix A: Mueller matrices}

The Mueller matrix for frame rotation from the $x$-axis towards the $y$-axis is ${ }^{28}$ :

$$
\mathrm{R}(\rho)=\left(\begin{array}{cccc}
1 & 0 & 0 & 0 \\
0 & \cos (2 \rho) & \sin (2 \rho) & 0 \\
0 & -\sin (2 \rho) & \cos (2 \rho) & 0 \\
0 & 0 & 0 & 1
\end{array}\right)
$$


The Mueller matrix for an ideal linear polarizer, whose transmission axis makes an angle $\rho$ with the $x$-axis is:

$$
M_{P}(\rho) \equiv R(-\rho) \frac{1}{2}\left(\begin{array}{llll}
1 & 1 & 0 & 0 \\
1 & 1 & 0 & 0 \\
0 & 0 & 0 & 0 \\
0 & 0 & 0 & 0
\end{array}\right) R(\rho)
$$

The Mueller matrix for an ideal linear retarder, whose fast axis makes an angle $\rho$ with the $x$-axis is:

$$
\mathrm{M}_{\mathrm{LR}}(\rho, \phi) \equiv \mathrm{R}(-\rho)\left(\begin{array}{cccc}
1 & 0 & 0 & 0 \\
0 & 1 & 0 & 0 \\
0 & 0 & \cos \phi & \sin \phi \\
0 & 0 & -\sin \phi & \cos \phi
\end{array}\right) \mathrm{R}(\rho)
$$

It follows that the Mueller matrix for an ideal quarter-wave plate is $\mathrm{M}_{\mathrm{QWP}}(\rho) \equiv \mathrm{M}_{\mathrm{LR}}\left(\rho, \frac{\pi}{2}\right)$.

${ }^{1}$ A. Loarte, B. Lipschultz, A. S. Kukushkin, G. F. Matthews, P. C. Stangeby, N. Asakura, G. F. Counsell, G. Federici, A. Kallenbach, K. Krieger, A. Mahdavi, V. Philipps, D. Reiter, J. Roth, J. Strachan, D. Whyte, R. Doerner, T. Eich, W. Fundamenski, A. Herrmann, M. Fenstermacher, P. Ghendrih, M. Groth, A. Kirschner, S. Konoshima, B. Labombard, P. Lang, A. W. Leonard, P. Monier-Garbet, R. Neu, H. Pacher, B. Pegourie, R. A. Pitts, S. Takamura, J. Terry, and E. Tsitrone, Nuclear Fusion 47 (2007), $10.1088 / 0029-5515 / 47 / 6 / \mathrm{S} 04$.

${ }^{2}$ W. Morris, J. R. Harrison, A. Kirk, B. Lipschultz, F. Militello, D. Moulton, and N. R. Walkden, IEEE Transactions on Plasma Science 46, 1217 (2018). ${ }^{3}$ J. Howard, Applied Optics 41, 197 (2002).

${ }^{4}$ J. Howard, C. Michael, F. Glass, and A. Danielsson, "Time-resolved twodimensional plasma spectroscopy using coherence-imaging techniques," (2003).

5 J. Howard, Journal of Physics B: Atomic, Molecular and Optical Physics 43 (2010), 10.1088/0953-4075/43/14/144010.

${ }^{6}$ M. Francon and S. Mallick, Polarization Interferometers (WileyInterscience, 1971).

${ }^{7}$ S. Davis, M. Abrams, and J. Brault, Fourier Transform Spectroscopy (Academic Press, 2001).

${ }^{8}$ X. Feng, A. Calcines, R. M. Sharples, B. Lipschultz, A. Perek, W. A. J. Vijvers, J. R. Harrison, J. S. Allcock, Y. Andrebe, B. P. Duval, and R. T. Mumgaard, Review of Scientific Instruments 92, 063510 (2021).

${ }^{9}$ S. A. Silburn, A Doppler Coherence Imaging Diagnostic for the Mega-Amp Spherical Tokamak, Ph.D. thesis, Durham University (2014).

${ }^{10}$ S. A. Silburn, J. R. Harrison, J. Howard, K. J. Gibson, H. Meyer, C. A. Michael, and R. M. Sharples, Review of Scientific Instruments 85 (2014), 10.1063/1.4891165.

${ }^{11}$ C. M. Samuell, J. D. Lore, W. H. Meyer, M. W. Shafer, S. L. Allen, T. E. Evans, and J. Howard, Physical Review Research 2, 1 (2020).

${ }^{12}$ V. Perseo, F. Effenberg, D. Gradic, R. König, O. P. Ford, F. Reimold, D. A. Ennis, O. Schmitz, and T. Sunn Pedersen, Nuclear Fusion 59 (2019), 10.1088/1741-4326/ab4320.

${ }^{13}$ D. Gradic, O. P. Ford, A. Burckhart, F. Effenberg, H. Frerichs, R. König, T. Lunt, V. Perseo, and R. C. Wolf, Plasma Physics and Controlled Fusion 60 (2018), 10.1088/1361-6587/aac4d2.

${ }^{14}$ T. Long, J. S. Allcock, L. Nie, R. M. Sharples, M. Xu, R. Ke, S. Zhang, S. A. Silburn, J. Howard, Y. Yu, B. Yuan, Z. H. Wang, X. M. Song, L. Liu, and X. R. Duan, Review of Scientific Instruments (2020), 10.1063/5.0005609.

${ }^{15}$ B. A. Lomanowski, A. G. Meigs, R. M. Sharples, M. Stamp, and C. Guillemaut, Nuclear Fusion 55 (2015), 10.1088/0029-5515/55/12/123028.

${ }^{16}$ J. R. Harrison, S. W. Lisgo, K. J. Gibson, P. Tamain, J. Dowling, and The Mast Team, in Journal of Nuclear Materials, Vol. 415 (2011).

${ }^{17}$ K. Verhaegh, B. Lipschultz, B. P. Duval, J. R. Harrison, H. Reimerdes, C. Theiler, B. Labit, R. Maurizio, C. Marini, F. Nespoli, U. Sheikh, C. K. Tsui, N. Vianello, and W. A. Vijvers, Nuclear Materials and Energy 12, 1112 (2017), arXiv:1607.04539.
${ }^{18}$ S. Potzel, R. Dux, H. W. Müller, A. Scarabosio, and M. Wischmeier, Plasma Physics and Controlled Fusion 56 (2014), 10.1088/07413335/56/2/025010.

${ }^{19}$ I. H. Hutchinson, Principles of Plasma Diagnostics arXiv:arXiv:1011.1669v3.

${ }^{20}$ O. Lischtschenko, K. Bystrov, G. De Temmerman, J. Howard, R. J. Jaspers, and R. König, Review of Scientific Instruments 81, 1 (2010).

${ }^{21}$ G. De Temmerman, M. A. Van Den Berg, J. Scholten, A. Lof, H. J. Van Der Meiden, H. J. Van Eck, T. W. Morgan, T. M. De Kruijf, P. A. Zeijlmans Van Emmichoven, and J. J. Zielinski, in Fusion Engineering and Design, Vol. 88 (2013) pp. 483-487.

${ }^{22}$ J. E. Millerd, N. J. Brock, J. B. Hayes, M. B. North-Morris, M. Novak, and J. C. Wyant, Interferometry XII: Techniques and Analysis 5531, 304 (2004).

${ }^{23}$ K. Creath and G. Goldstein, Biomedical Optics Express 3, 2866 (2012).

${ }^{24}$ T. Tahara, R. Yonesaka, S. Yamamoto, T. Kakue, P. Xia, Y. Awatsuji, K. Nishio, S. Ura, T. Kubota, and O. Matoba, IEEE Journal on Selected Topics in Quantum Electronics 18, 1387 (2012).

${ }^{25}$ K. Ishikawa, K. Yatabe, N. Chitanont, Y. Ikeda, Y. Oikawa, T. Onuma, H. Niwa, and M. Yoshii, Optics Express (2016), 10.1364/oe.24.012922.

${ }^{26}$ W. H. Steel, Interferometry, 2nd ed. (Cambridge University Press, 1987).

${ }^{27}$ M. P. Kothiyal and C. Delisle, Applied Optics 24, 4439 (1985).

${ }^{28}$ R. A. Chipman, W.-S. T. Lam, and G. Young, Polarized Light and Optical Systems (2018).

${ }^{29}$ C. A. Michael, J. Howard, and B. D. Blackwell, Physics of Plasmas (2004), $10.1063 / 1.1768175$.

30 “https://www.sony-semicon.co.jp/e/products/IS/industry/product/polarization.html,".

${ }^{31}$ F. E. Veiras, L. I. Perez, and M. T. Garea, Applied Optics 49, 2769 (2010).

${ }^{32} \mathrm{~B}$. Kimbrough and J. Millerd, Interferometry XV: Techniques and Analysis 7790, 77900K (2010).

${ }^{33}$ M. Servin, J. A. Quiroga, and M. Padilla, Fringe Pattern Analysis for Optical Metrology: Theory, Algorithms, and Applications (Wiley-VCH, 2014) pp. $177-186$.

${ }^{34}$ C. M. Samuell, S. L. Allen, W. H. Meyer, and J. Howard, Journal of Instrumentation 12 (2017), 10.1088/1748-0221/12/08/C08016.

${ }^{35} \mathrm{P}$. Urlings, "Multiple Delay Coherence Imaging Charge Exchange Recombination Spectroscopy (MSc thesis)," Tech. Rep. (Eindhoven University of Technology, 2015).

${ }^{36}$ D. J. Bone, H.-A. Bachor, and R. J. Sandeman, Applied Optics 25, 1653 (1986).

${ }^{37}$ C. Bowman, J. R. Harrison, B. Lipschultz, S. Orchard, K. J. Gibson, M. Carr, K. Verhaegh, and O. Myatra, Plasma Physics and Controlled Fusion 62 (2020), 10.1088/1361-6587/ab759b.

${ }^{38}$ J. Rosato, Y. Marandet, and R. Stamm, Journal of Quantitative Spectroscopy and Radiative Transfer 187, 333 (2017).

${ }^{39}$ S. Trippe, Journal of the Korean Astronomical Society 47, 15 (2014), arXiv:1401.1911.

${ }^{40}$ J. Rosato, "Private communication,".

${ }^{41}$ A. E. Shumack, V. P. Veremiyenko, D. C. Schram, H. J. De Blank, W. J. Goedheer, H. J. Van Der Meiden, W. A. Vijvers, J. Westerhout, N. J. Lopes Cardozo, and G. J. Van Rooij, Physical Review E - Statistical, Nonlinear, and Soft Matter Physics (2008), 10.1103/PhysRevE.78.046405.

${ }^{42}$ D. S. Sivia and J. Skilling, Data Analysis: A Bayesian Tutorial (Oxford University Press, 2006).

${ }^{43}$ H. J. Van Der Meiden, A. R. Lof, M. A. Van Den Berg, S. Brons, A. J. Donné, H. J. Van Eck, P. M. Koelman, W. R. Koppers, O. G. Kruijt, N. N. Naumenko, T. Oyevaar, P. R. Prins, J. Rapp, J. Scholten, D. C. Schram, P. H. Smeets, G. Van Der Star, S. N. Tugarinov, and P. A. Van Emmichoven, Review of Scientific Instruments 83 (2012), 10.1063/1.4768527.

${ }^{44}$ J. Leland, S. Elmore, A. Kirk, H. J. van der Meiden, J. Scholten, S. Y. Allan, and J. W. Bradley, Nuclear Materials and Energy 27, 100954 (2021).

${ }^{45}$ C. Costin, V. Anita, F. Ghiorghiu, G. Popa, G. De Temmerman, M. A. Van Den Berg, J. Scholten, and S. Brons, Plasma Sources Science and Technology 24 (2015), 10.1088/0963-0252/24/1/015014.

${ }^{46}$ G. R. Akkermans, I. G. Classen, R. Perillo, H. J. Van Der Meiden, F. Federici, and S. Brezinsek, Physics of Plasmas 27 (2020), 10.1063/5.0017714.

${ }^{47}$ A. H. Andersen and A. C. Kak, Ultrasonic Imaging (1984), $10.1177 / 016173468400600107$. 